\title{
Article
}

Subscriber access provided by University of East Anglia Library

\section{Structural and Biochemical Insights into Dimethylsulfoniopropionate Cleavage by Cofactor-bound DddK from the Prolific Marine Bacterium Pelagibacter}

Nicholas J. Schnicker, Saumya M. De Silva, Jonathan D. Todd, and Mishtu Dey

Biochemistry, Just Accepted Manuscript • Publication Date (Web): 16 May 2017

Downloaded from http://pubs.acs.org on May 17, 2017

\section{Just Accepted}

"Just Accepted" manuscripts have been peer-reviewed and accepted for publication. They are posted online prior to technical editing, formatting for publication and author proofing. The American Chemical Society provides "Just Accepted" as a free service to the research community to expedite the dissemination of scientific material as soon as possible after acceptance. "Just Accepted" manuscripts appear in full in PDF format accompanied by an HTML abstract. "Just Accepted" manuscripts have been fully peer reviewed, but should not be considered the official version of record. They are accessible to all readers and citable by the Digital Object Identifier (DOI®). "Just Accepted" is an optional service offered to authors. Therefore, the "Just Accepted" Web site may not include all articles that will be published in the journal. After a manuscript is technically edited and formatted, it will be removed from the "Just Accepted" Web site and published as an ASAP article. Note that technical editing may introduce minor changes to the manuscript text and/or graphics which could affect content, and all legal disclaimers and ethical guidelines that apply to the journal pertain. ACS cannot be held responsible for errors or consequences arising from the use of information contained in these "Just Accepted" manuscripts. 


\title{
Structural and Biochemical Insights into Dimethylsulfoniopropionate \\ Cleavage by Cofactor-bound DddK from the Prolific Marine Bacterium \\ Pelagibacter $^{\$}$
}

\author{
Nicholas J. Schnicker ${ }^{1,3}$, Saumya M. De Silva ${ }^{1,3}$, Jonathan D. Todd ${ }^{2}$, and Mishtu Dey ${ }^{1 *}$ \\ ${ }^{1}$ Department of Chemistry, The University of Iowa, Iowa City, IA 52242. \\ ${ }^{2}$ School of Biological Sciences, University of East Anglia, Norwich Research Park, United \\ Kingdom. \\ ${ }^{3}$ Authors contributed equally to the work.
}

Running Title: DddK exhibits promiscuity in metal recognition for DMSP catabolism.

*To whom correspondence should be addressed. Mishtu Dey, Department of Chemistry, University of Iowa, W285 Chemistry Building, Iowa City, IA 52242-1727. Tel: 319-3841319. Fax: 319-335-1270.E-mail: mishtu-dey@uiowa.edu.

${ }^{\$}$ This work was supported by University of Iowa College of Liberal Arts and Sciences.

The authors declare no conflict of interest.

X-ray crystallography, atomic coordinates, and structure factors of have been deposited in the Protein Data Bank, www.pdb.org (PDB ID codes 5TFZ and 5TGO). 


\begin{abstract}
Abbreviations: DMSP, dimethylsulfoniopropionate; DddK, a DMSP lyase; DMS, dimethylsulfide; P. ubique, Pelagibacter ubique; DSBH, double-stranded beta-helix.
\end{abstract}

Keywords: Dimethylsulfoniopropionate, DMSP lyase DddK, SAR 11 bacteria, dimethylsulfide, acrylate, crystal structure, cupin-fold, metalloenzyme, enzyme kinetics. 


\begin{abstract}
Enormous amounts of the organic osmolyte dimethylsulfoniopropionate (DMSP) are produced in marine environments where bacterial DMSP lyases cleave it yielding acrylate and the climate-active gas dimethylsulfide (DMS). SAR11 bacteria are the most abundant clade of heterotrophic bacteria in the oceans and they play a key role in DMSP catabolism. An important environmental factor affecting DMS generation via DMSP lyases is the availability of metal ions since they are essential cofactors for many of these enzymes. Here we examine the structure and activity of DddK in the presence of various metal ions. We have established that DddK containing a double stranded $\beta$-helical motif utilizes various divalent metal ions as cofactors for catalytic activity. However, nickel, an abundant metal ion in marine environments, adopts a distorted octahedral coordination environment and conferred the highest DMSP lyase activity. Crystal structures of cofactor bound DddK reveal key metal ion binding and catalytic residues and provide the first rationalization for varying activities with different metal ions. The structures of DddK along with site-directed mutagenesis and UV-visible studies are consistent with Tyr 64 acting as a base to initiate the $\beta$-elimination reaction of DMSP. Our biochemical and structural studies provide a detailed understanding of DMS generation by one of the ocean's most prolific bacterium.
\end{abstract}


Generated in marine environments at $\sim 10^{9}$ tons annually, the organosulfur compound dimethylsulfoniopropionate (DMSP) is an osmolyte and antioxidant produced by many marine phytoplankton and macroalgae, where it can accumulate to very high $(\sim 0.5 \mathrm{M})$ intracellular concentrations. ${ }^{1-5}$ When released into the environment, DMSP is an important solute for marine microbial communities that import and catabolize it via two main pathways. $^{4-6}$ It was believed that the majority of DMSP in seawater is catabolized via the demethylation pathway, common to many marine roseobacters and the highly abundant SAR11 bacteria. This pathway involves a series of enzymes producing acetaldehyde and methanethiol (MeSH), an important microbial reduced sulfur source. ${ }^{6,7}$ However, this study focuses on the DMSP cleavage pathway where DMSP is cleaved by enzymes known as "DMSP lyases" to generate acrylate or hydroxypropionate, and $\sim 10^{7}$ tons of dimethyl sulfide (DMS) per annum. ${ }^{4,8}$ DMSP and DMS have important roles in marine microbial food webs and in global sulfur cycling. DMS is a signaling molecule for some zooplankton, crustaceans, and seabirds and is potentially involved in climate regulation since DMS oxidation products are cloud condensation nuclei. ${ }^{1-4}$ Furthermore, the other DMSP cleavage products, acrylate and hydroxypropionate, are compounds of industrial importance.

Despite the environmental and industrial significance of the DMSP cleavage products acrylate and DMS, the biochemistry of the DMSP lyases is relatively understudied. There are seven distinct functional DMSP lyase enzymes identified in proteobacteria and some ascomycete fungi (DddD, DddY, DddP, DddL, DddQ, DddW and DddK), and, Alma1, only currently found in phytoplankton. ${ }^{9-11}$ While Almal (Asp/Glu/hydantoin racemase), DddD (class III CoA-transferase), DddP (M24B metallopeptidase family), and DddY (periplasmic protein with no conserved protein domains) are from unrelated protein families, the remaining lyases DddL, DddQ, DddW, and the recently identified DddK from Pelagibacter 
ubique (P. ubique) HTCC1062, belong to the cupin superfamily of enzymes, which are the subject of this study.

The cupin superfamily is considered one of the most functionally diverse protein superfamilies known to exist and all members contain a C-terminal Greek-key (or, cupin) domain, which is characterized by a double stranded $\beta$-helix (DSBH) fold containing two conserved sequence motifs, $\mathrm{GX}_{5} \mathrm{HXHX}_{3,4} \mathrm{E}$ (or D) $\mathrm{X}_{6} \mathrm{G}$ and $\mathrm{GX}_{5} \mathrm{PXGX}_{2} \mathrm{HX}_{3} \mathrm{~N}$. $^{12}$ These conserved motif regions contain residues in the active site that are involved in binding diverse metal ions, such as iron, manganese, nickel, cobalt, and zinc. ${ }^{13}$ However, only specific metallocofactors influence the interactions of the enzyme with the substrate for efficient catalysis. ${ }^{12,14-16}$ A number of different ligand coordination combinations exist with the most common being 2/3-His and 1-carboxylate (Asp/Glu), and less frequently 2-His/1Glu/1-Tyr, 2-His/1-Glu/1-Gln, or, 3-His/1-Gln. ${ }^{15}$

The metallocofactor specificity of cupin-fold containing DMSP lyases is unclear and the mechanism of DMSP cleavage is not fully established, which are primarily due to the lack of sufficient biochemical and structural data. Biochemical studies revealed that the Ruegeria pomeroyi DSS-3 DMSP lyase DddW has highest enzyme activities with Fe(II) or $\mathrm{Mn}(\mathrm{II})$ as cofactors, ${ }^{17}$ but the crystal structure of DddW has not been determined. The crystal structure of DMSP lyase DddQ, from R. lacuscaerulensis, showed that the as-isolated enzyme contains a DSBH fold and uses 3-His, 1-Glu, and 1-Tyr to bind the central zinc ion at its active site. ${ }^{18}$ DddQ was reported as a $\mathrm{Zn}(\mathrm{II})$-dependent enzyme based on crystal structure and atomic absorption spectral results showing $42 \%$ zinc present in the isolated protein. However, the same study found that excess zinc inhibited DddQ activity and that DddQ was most active in the presence of $\mathrm{Co}(\mathrm{II})$ and $\mathrm{Mn}(\mathrm{II})$, but other relevant metal ions, such as iron and nickel were not investigated. ${ }^{18}$ Recent kinetic and structural studies examining the importance of iron in DddQ-catalyzed DMSP cleavage revealed that both Fe(III)-oxidized 
and $\mathrm{Fe}(\mathrm{II})$-reduced species are active. ${ }^{19}$ The slow turnover rates $\left(k_{\text {cat }} / K_{m}\right.$ of $\left.0.3-0.7 \mathrm{M}^{-1} \mathrm{~s}^{-1}\right)$ for iron-bound DddQ indicate that iron may not be the most catalytically active metal ion for DddQ when compared to the mononuclear iron-dependent DddW $\left(k_{c a t} / K_{m}\right.$ of $2.1 \times 10^{3} \mathrm{M}^{-1} \mathrm{~s}^{-}$ $\left.{ }^{1}\right)$. The crystal structure of Fe(III)-bound DddQ revealed conformational changes associated with DMSP binding. Based on the structures of DddQ, two mechanisms of DMSP cleavage have been proposed that invoke different tyrosine residues acting as a Lewis base to initiate catalysis. Furthermore, biochemical and structural studies of DddP led to the proposal of an ion-shift mechanism involving a diiron center, but the specific activity for iron-bound DddP $\left(0.31 \mu \mathrm{mol}\right.$ of DMS $\min ^{-1} \mathrm{mg}$ of $\left.\mathrm{DddP}^{-1}\right)$ is 200 times lower than that of Fe(II)-DddW (65 $\mu \mathrm{mol}$ of acrylate $\min ^{-1} \mathrm{mg}$ of $\mathrm{DddW}^{-1}$ ). The crystal structure of a catalytically efficient DMSP lyase is not available and therefore, obtaining this highly important structure will provide insight into the bacterial cleavage of DMSP to DMS by a lyase of the cupin superfamily.

To gain mechanistic insight into environmentally relevant DMSP cleavage by cupin-domain containing DMSP lyases we sought to investigate the recently discovered DddK from SAR11 bacteria, which are the most abundant and ubiquitous clade of heterotrophic bacteria in the oceans, often comprising $30 \%$ of the surface microbial community. ${ }^{9,20}$ A multiple sequence alignment of DddK with DddL, DddQ, and DddW shows conserved active site residues required for metallocofactor binding (Figure 1). Here we over-express and purify P. ubique HTCC1062 DddK and present the first crystal structure of a catalytically competent DMSP lyase with its $\mathrm{Ni}(\mathrm{II})$ cofactor. The structures of the Ni- and diacrylate-bound enzyme and the mixed $\mathrm{Fe}(\mathrm{III}) / \mathrm{Zn}$ (II)-bound DddK show the presence of the DSBH fold and reveal key residues involved in both metal ion binding and DMSP catabolism. The metal binding ability and catalytic activity of DddK were examined in the presence of various metal ions. DddK displayed promiscuous binding to various metal ions, but exhibited preference for select divalent transition metal ions, such as $\mathrm{Ni}(\mathrm{II})$ in catalyzing the DMSP cleavage reaction. 
Furthermore, site-directed mutagenesis and UV-visible studies have confirmed distinct metal ion binding and catalytic roles of His 56 , His 58, Glu 62, Tyr 64, His 96, and Tyr 122. Given the recent discovery that DMSP cleavage converted almost $60 \%$ of intracellular DMSP to DMS in Pelagibacter and the global abundance of SAR11 bacteria, ${ }^{9}$ our findings on the mechanism of Ni(II)-DddK catalyzed DMSP cleavage provide a greater understanding of an enzyme that is potentially very important in global DMS production.

Figure 1. Sequence alignment of $P$. ubique DddK (PuDddK), R. pomeroyi DddW (RpDddW), Rhodobacter sphaeroides DddL (RsDddL), and R. lacuscaerulensis DddQ (RIDddQ) with secondary structures defined above the sequence. $\alpha$-helices are indicated by helical lines (not labeled), $\beta$-strands are indicated by arrows with $\mathrm{B}$ labels, and a roman numeral after the B indicates a $\beta$-strand in the cupin fold. Active site residues are highlighted in red. Catalytically relevant tyrosines in DddK are highlighted in green. Similar residues are in black bold characters and boxed in yellow. The secondary structure elements are from NiDddK (5TFZ) and DddQ (5JSO). The figure was made using ESPript 3.

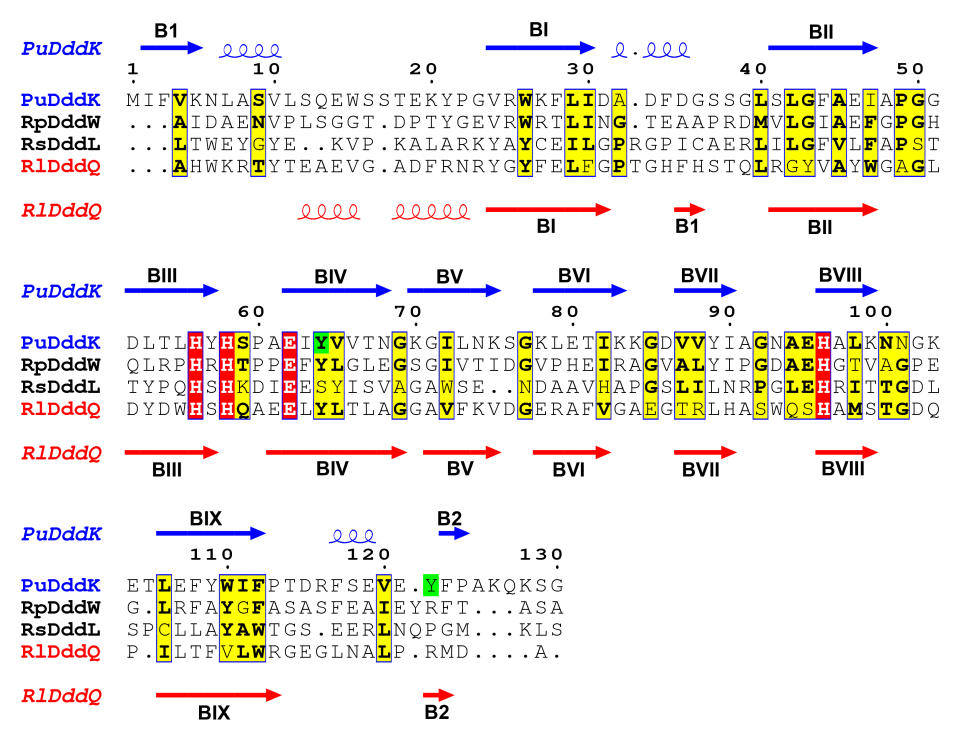

\section{Materials and Methods}

Protein Expression and Purification. The $d d d K$ gene from SAR11 strain "Candidatus Pelagibacter ubique HTCC1062 was optimized for expression in E. coli and cloned into the pET28a expression vector (Novagen) using restriction sites $N d e \mathrm{I}$ and BamHI to include an Nterminal 6 histidine tag. The DddK plasmid was transformed into E. coli BL21 DE3 cells (Life Technologies, Grand Island, NY). Cells were grown in LB media at $37{ }^{\circ} \mathrm{C}$ until cultures 
reached an optical density of 0.6 at $600 \mathrm{~nm}$. The temperature was lowered to $25{ }^{\circ} \mathrm{C}$ and the cells were induced with $0.5 \mathrm{mM}$ isopropyl-D-thiogalactopyranoside and grown for 6 hours before harvesting by centrifugation at $4000 \mathrm{rpm}$ at $4{ }^{\circ} \mathrm{C}$. Cells were re-suspended in lysis buffer (50 mM HEPES pH 7.5, $300 \mathrm{mM} \mathrm{NaCl}, 5 \mathrm{mM}$ imidazole) along with phenylmethylsulfonyl fluoride. The cell suspension was sonicated for 2 min total time with 4 seconds 'on' and 15 seconds 'off'. The cell lysate was centrifuged at $20000 \mathrm{rpm}$ at $4{ }^{\circ} \mathrm{C}$ for $30 \mathrm{~min}$ and the soluble fraction was loaded onto a column packed with $5 \mathrm{~mL}$ of $\mathrm{Ni}(\mathrm{II})$ nitrilotriacetic acid agarose (MCLAB, San Francisco, CA) pre-equilibrated in lysis buffer. The column was washed with $100 \mathrm{~mL}$ of lysis buffer containing $30 \mathrm{mM}$ imidazole before eluting the protein in lysis buffer containing $500 \mathrm{mM}$ imidazole. Fractions were analyzed by SDS-PAGE, pooled, concentrated, and loaded onto a Superdex 200 16/60 column (120 mL, GE Healthcare) pre-equilibrated in storage buffer (50 mM HEPES pH 7.5 and $100 \mathrm{mM}$ $\mathrm{NaCl}$ ). Fractions $>99 \%$ purity based on SDS-PAGE were pooled and concentrated to 40 $\mathrm{mg} / \mathrm{mL}$; aliquots were stored at $-80{ }^{\circ} \mathrm{C}$ until use. The final yield was $25 \mathrm{mg}$ protein $/ \mathrm{g}$ cell pellet. The DddK variants Y64A and Y122A were prepared using PCR methods and verified by DNA sequencing. The variant proteins were expressed and purified as above. The final yield of purified Y64A variant (1.24 mg protein/g cell pellet) was significantly lower than either wild-type and Y122A (19 mg protein/g cell pellet).

Preparation of apo-DddK. Concentrated as-isolated wild-type DddK and Tyr64Ala, Tyr122Ala DddK variants were dialyzed into a chelation buffer (50 mM MES pH 6, $100 \mathrm{mM}$ $\mathrm{NaCl}, 5 \mathrm{mM}$ EDTA) to remove any metals bound to the protein. The chelation buffer was exchanged eight times over the course of 4-5 days. After metal chelation, the protein was concentrated using an Amicon Ultra 10,000 MWCO membrane (Millipore, Billerica, MA) and then exchanged into the storage buffer. The metal content of as-isolated and apo-DddK was confirmed by inductively coupled plasma optical emission spectroscopy (ICP-OES). 
Metal analysis. Protein samples were made to a final concentration of $20 \mu \mathrm{M}$ for metal analysis by ICP-OES. Samples were prepared in a solution containing $8 \%$ protein in storage buffer, $90 \%$ water, and $2 \%$ metal-free nitric acid. Samples were analyzed for the following metals: $\mathrm{Mn}, \mathrm{Fe}, \mathrm{Co}, \mathrm{Ni}, \mathrm{Cu}$, and $\mathrm{Zn}$ as previously described. ${ }^{17}$

UV-visible spectroscopy. UV-visible spectra were measured using a Beckman Coulter DU 720 UV-Vis spectrophotometer (Brea, CA). The spectrum of as-isolated DddK $(1.2 \mathrm{mM})$ in $50 \mathrm{mM}$ HEPES pH 7.5 and $100 \mathrm{mM} \mathrm{NaCl}$ was recorded from 400-800 nm. Excess dithionite $(5 \mathrm{mM})$ was added to the as-isolated enzyme and the spectrum was collected again. The spectra of as-isolated DddK variants Y64A (1.2 mM) and Y122A (1.2 mM) were recorded similar to that of wild type DddK. The UV-visible spectrum of Ni(II)-bound DddK was collected on the same instrument from $400-1100 \mathrm{~nm}$ using $1 \mathrm{mM}$ apo-DddK in the presence of $1 \mathrm{mM} \mathrm{NiCl}_{2}$.

DMSP lyase activity and metal binding assays. Assays to determine the optimal activity of DddK were done using $10 \mu \mathrm{M}$ of apo-enzyme (monomer concentration) in $50 \mathrm{mM}$ Tris $\mathrm{pH} 8.5$ and $100 \mathrm{mM} \mathrm{NaCl}$ at $20{ }^{\circ} \mathrm{C}$ with $20 \mathrm{mM}$ DMSP, in addition to a lower concentration $(2 \mathrm{mM})$. Activity assays were performed at varying concentrations of metal ions to determine the binding affinities and metal preferences. DddK was incubated separately with each metal ion for 30 minutes prior to addition of DMSP. The initial velocities were measured for 2 minutes by tracking the production of acrylate as previously described. ${ }^{17}$ Incubation and activity measurements for $\mathrm{Fe}(\mathrm{II})$ were done under nitrogen atmosphere. The binding affinity of Fe(III) to DddK could not be determined because the product acrylate was not observed on HPLC, indicating that the presence of Fe(III) did not activate the enzyme. Determination of kinetic parameters and DMSP dependence of DddK activity was performed in the presence of $60 \mu \mathrm{M}$ Ni(II) using $10 \mu \mathrm{M}$ DddK. 
Data analysis and equations. Data were fit with SigmaPlot (Systat Software Inc., Point Richmond, CA). The DMSP dependence of DddK activity was fit to the Michaelis-Menten equation as reported previously. ${ }^{17,19}$ The $K_{d}$ values for metal binding to DddK in Figure S2 were calculated by fitting the data to Eq. (1), which was modified to include the Hill coefficient. $^{17,21-23}$

$$
V=V_{o}+\left(V_{m}-V_{o}\right) \frac{\left(n P+x^{h}+K_{d}^{h}\right)-\sqrt[2]{\left(n P+x^{h}+K_{d}^{h}\right)^{2}-4 n P x^{h}}}{2 n P}
$$

where, $V$ is the measured rate resulting from the binding of a metal ion to apo-DddK, $V_{0}$ is the lowest rate measured, $V_{m}$ is the maximum activity measured, $K_{d}$ is the dissociation constant, $\mathrm{P}$ and $\mathrm{x}$ are total protein and total metal ion concentrations respectively, $\mathrm{h}$ is the Hill coefficient, and $\mathrm{n}$ is the number of binding sites. The fractional rate is obtained by converting the measured rates with $\mathrm{V}-\mathrm{V}_{\mathrm{o}} / \mathrm{V}_{\max }-\mathrm{V}_{\mathrm{o}}$.

Crystallization. Purified DddK was concentrated in 50 mM HEPES pH 7.5 containing $100 \mathrm{mM} \mathrm{NaCl}$ to two different concentrations for crystallization trials. Crystals were grown using sitting-drop vapor-diffusion at $20{ }^{\circ} \mathrm{C}$ with initial hits obtained from sparse-matrix screening (Hampton Research, Emerald Biosystems).

Crystallization of Ni-DddK was accomplished using $1 \mu \mathrm{L}$ of a $20 \mathrm{mg} / \mathrm{mL}$ solution of as-isolated DddK mixed with $1 \mu \mathrm{L}$ of precipitant solution containing $0.1 \mathrm{M}$ potassium thiocyanate and 30\% PEG 2000 MME. While excess nickel was not used during crystallization, crystals of Ni-bound DddK were rod shaped and grew in about 6 days. Crystals were soaked in the precipitant solution containing $50 \mathrm{mM}$ acrylate for 5 hours. For cryocooling, crystals were looped and washed in the precipitant solution containing $20 \%$ glycerol and $50 \mathrm{mM}$ acrylate for $\sim 5$ minutes and then submerged in liquid nitrogen. While attempting to soak the product acrylate into the crystal, diacrylate was later determined to be 
the contaminating species bound in the structure solved. The presence of diacrylate was confirmed by mass spectrometry as discussed below.

Crystallization of FeZn-DddK was achieved using $1 \mu \mathrm{L}$ of a $40 \mathrm{mg} / \mathrm{mL}$ solution of asisolated DddK with $1 \mu \mathrm{L}$ of precipitant solution containing 0.1 M HEPES pH 7.5, 0.2 M $\mathrm{NaCl}$, and 20\% PEG 3000. Crystals of FeZn-DddK were rod shaped and grew in about 2 days. For cryocooling, crystals were looped and washed in the precipitant solution containing $20 \%$ glycerol for $\sim 5$ minutes and then submerged in liquid nitrogen.

X-ray Data Collection and Structure Determination. The data set for Ni-DddK was collected at $100 \mathrm{~K}$ at the Advanced Light Source, beamline 4.2.2. Data collection parameters were: $0.2^{\circ}$ oscillation, $0.3 \mathrm{~s}$ exposure time, and a $300 \mathrm{~mm}$ detector distance. A fluorescence emission scan on the crystal used for data collection confirmed the presence of nickel with a peak at $7.48 \mathrm{keV}$. The data set for FeZn-DddK was collected at $100 \mathrm{~K}$ at the Advanced Photon Source, beamline 19-ID-D. Data collection parameters were: $0.5^{\circ}$ oscillation, $0.5 \mathrm{~s}$ exposure time, and a $200 \mathrm{~mm}$ detector distance. A fluorescence emission scan on the crystal gave peaks for iron and zinc at 6.40 and $8.64 \mathrm{keV}$, respectively. The data were indexed and integrated using the XDS package ${ }^{24}$ and merged and scaled using AIMLESS. ${ }^{25}$ Data collection and refinement statistics are summarized in Table 1.

Table 1. Data Collection and Refinement Statistics.

\begin{tabular}{|l|l|l|}
\hline & Ni-DddK & FZ-DddK \\
\hline Data Collection & & \\
\hline PDB code & 5 TFZ & 5 TGO \\
\hline Space Group & P $2_{1} 2_{1} 2_{1}$ & C $222_{1}$ \\
\hline Unit Cell Dimensions & & \\
\hline a b c $(\AA)$ & 43.454 .7118 .3 & 55.185 .454 .4 \\
\hline$\alpha \beta \gamma($ deg$)$ & 909090 & 909090 \\
\hline Beam line & ALS 4.2.2 & APS 19-ID-D \\
\hline Wavelength $(\AA)$ & 1.00 & 0.979 \\
\left.\hline${\text { Mosaicity }\left({ }^{\circ}\right)}^{(}\right)$ & 0.29 & 0.24 \\
\hline Resolution Range $(\AA)^{\mathrm{a}}$ & $59.17-2.20$ & $46.28-1.44$ \\
\hline Observations $^{\text {a }}$ & $(2.32-2.20)$ & $(1.52-1.44)$ \\
\hline Unique Reflections $^{\mathrm{a}}$ & $98706(11900)$ & $140675(14384)$ \\
\hline
\end{tabular}




\begin{tabular}{|c|c|c|}
\hline Redundancy $^{\mathrm{a}}$ & $6.6(5.6)$ & $6.0(4.3)$ \\
\hline Completeness $(\%)^{\mathrm{a}}$ & $100(99.9)$ & $99.6(97.6)$ \\
\hline $\mathrm{I} / \sigma(\mathrm{I})^{\mathrm{a}}$ & $9.4(2.8)$ & $29.8(7.2)$ \\
\hline $\mathrm{R}_{\text {merge }}(\%)^{\mathrm{a}, \mathrm{b}}$ & $13.8(55.8)$ & $3.1(15.2)$ \\
\hline $\mathrm{R}_{\mathrm{pim}}(\%)^{\mathrm{a}, \mathrm{c}}$ & $6.3(28.1)$ & $1.5(8.8)$ \\
\hline $\mathrm{CC}_{1 / 2}$ & $0.994(0.844)$ & $0.999(0.971)$ \\
\hline \multicolumn{3}{|l|}{ Refinement } \\
\hline $\mathrm{R}_{\text {work }} \mathrm{R}_{\text {free }}{ }^{\mathrm{d}}(\%)$ & $17.2(22.4)$ & $11.0(14.0)$ \\
\hline Resolution Range $(\AA)$ & $59.2-2.20$ & $27.5-1.44$ \\
\hline No. of Reflections & 27455 & 44346 \\
\hline No. Molecules per asu ${ }^{\mathrm{c}}$ & 2 & 1 \\
\hline \multicolumn{3}{|l|}{ Number of Atoms: } \\
\hline Protein & 1992 & 2297 \\
\hline $\mathrm{Ni}$ & 2 & - \\
\hline $\mathrm{Fe}$ & - & 1 \\
\hline$\overline{\mathrm{Zn}}$ & - & 1 \\
\hline Diacrylate & 20 & - \\
\hline Water & 140 & 172 \\
\hline \multicolumn{3}{|l|}{ Average B-Factor $\left(\AA^{2}\right)$} \\
\hline Protein & 25.3 & 17.2 \\
\hline $\mathrm{Ni}$ & 33.0 & - \\
\hline $\mathrm{Fe}$ & - & 10.2 \\
\hline $\mathrm{Zn}$ & - & 9.0 \\
\hline Diacrylate & 49.9 & - \\
\hline Water & 34.4 & 30.7 \\
\hline RMSD Bond Lengths $(\AA)$ & 0.011 & 0.010 \\
\hline RMSD Bond Angles (deg) & 1.08 & 1.11 \\
\hline Ramachandran favored (\%) & 98.0 & 98.0 \\
\hline Ramachandran allowed (\%) & 2.0 & 2.0 \\
\hline Clash score & 6.4 & 3.0 \\
\hline
\end{tabular}

${ }^{\mathrm{a}}$ Values in parentheses are the highest-resolution shell. ${ }^{\mathrm{b}} \mathrm{R}_{\mathrm{sym}}=\sum_{\mathrm{hkl}} \sum_{\mathrm{i}} \mathrm{I}_{\mathrm{i}}(\mathrm{hkl})-<\mathrm{I}(\mathrm{hkl})>\mid / \sum_{\mathrm{hkl}} \sum_{\mathrm{i}} \mathrm{I}_{\mathrm{i}}(\mathrm{hkl})$, where $\mathrm{I}_{\mathrm{i}}(\mathrm{hkl})$ is the $\mathrm{i}^{\text {th }}$ measured diffraction intensity and $<\mathrm{I}(\mathrm{hkl})>$ is the mean intensity for the miller index (hkl). ${ }^{c} \mathrm{R}_{\text {factor }}=\sum_{\text {hkl }}|| \mathrm{F}_{\mathrm{o}}(\mathrm{hkl})|-| \mathrm{F}_{\mathrm{c}}(\mathrm{hkl})|| / \sum_{\mathrm{hkl}}\left|\mathrm{F}_{\mathrm{o}}(\mathrm{hkl})\right| .{ }^{\mathrm{d}} \mathrm{R}_{\text {free }}=\mathrm{R}_{\text {factor }}$ for a test set of reflections (5\%). ${ }^{\mathrm{e}}$ asu, asymmetric unit.

Molecular replacement was done using PHASER ${ }^{26}$ and 5TVE was used as a starting model. Model building and addition of ligands and waters was done using COOT. Refinement was carried out using phenix.refine from the PHENIX software package. ${ }^{27}$ Structures containing metal ions had bond length and angle parameters generated by phenix.metal_coordination. Default refinement parameters in PHENIX were used, in addition to anomalous refinement. In Ni-DddK, TLS refinement was also used and TLS groups were defined automatically by phenix.refine. In FeZn-DddK, the protein atoms and the metals 
were refined anisotropically while waters and hydrogens were treated as isotropic. The hydrogens were refined as riding hydrogens. Due to the complex nature of the active site in FeZn-DddK, the occupancies for each metal and its corresponding coordinated water molecule and Glu 62 conformation were linked. Following several cycles of manual model building and refinement, ligands were modeled into clear remaining electron density guided by simulated annealing OMIT maps. Anomalous maps were generated using phenix.maps to assist in the placement of metal ions in the active sites. Software used in this project was curated by SBGrid. ${ }^{28}$ Both structures contain missing residues from the $\mathrm{N}$ - and $\mathrm{C}$-termini, which varies slightly for each model and chain. The missing residues for each structure and chain are as follows: FeZn-DddK, -19 to -7 , Ni-DddK, chain A: -19 to -1 and 127-130, chain B: -19 to -5 and $127-130$. The structures were validated using the wwPDB validation server, ${ }^{29}$ MOLPROBITY, ${ }^{30}$ and the CheckMyMetal server. ${ }^{31}$ All structural figures were made using PyMOL.

Identification of Diacrylate by Mass Spectrometry. Mass spectrometry data was collected on a Waters Q-Tof Premier using electrospray ionization in negative ion mode. Analysis was done on samples from a stock bottle of acrylic acid that was used in crystallization experiments. Peaks less than $1 \%$ of the relative peak intensity were subtracted from the displayed spectrum. Based on the ratio of intensities of acrylate to diacrylate, diacrylate accounted for $28 \%$.

\section{Results and Discussion}

\section{Metal content and Spectral Properties of as-isolated DddK from Pelagibacter ubique} HTCC1062. Metal analysis indicated that purified as-isolated DddK ( MW $16.5 \mathrm{kDa})$ consists of $40 \%$ iron, $18 \%$ zinc, $7 \%$ nickel, and $4 \%$ manganese per monomer (Figure S1A). As-isolated DddK was violet color in solution and exhibited an absorption maximum at $\sim 530$ 
$\mathrm{nm}$ (Figure 2A). A peak around this wavelength has been observed for DddQ ${ }^{19}$ and other enzymes 32,33 that exhibit a Fe(III)-tyrosinate ligand-to-metal charge transfer (LMCT) transition. Addition of dithionite to DddK diminished the intensity of the $530 \mathrm{~nm}$ band, consistent with oxidized Fe(III) species being responsible for the absorption (Figure 2A). In order to establish which Tyr residue was contributing to the LMCT band, alanine variants of tyrosine residues located in the active site (Y64A and Y122A) were generated. While Y122A showed an absorbance maximum at $\sim 560 \mathrm{~nm}$ with reduced intensity, Y64A did not exhibit any absorbance in this region (Figure 2A), thus confirming that Tyr 64 is likely responsible for the Fe(III)-tyrosinate LMCT in DddK.

Figure 2. Catalytic and spectral properties of DddK. (A) UV-visible spectra of $1.2 \mathrm{mM}$ asisolated DddK (solid line), dithionite reduced DddK (dotted line), DddK variants Y122A (dashed line), DddK variants Y64A (dash-dot line). (B) Effects of various transition metal ions $(80 \mu \mathrm{M})$ on the activity of apo-DddK. (C) Activity of as-isolated Y64A and Y122A DddK variants compared to WT DddK. (D) DMSP dependence of Ni(II)-DddK activity, $K_{m}$ : $5.13 \pm 0.4 \mathrm{mM}$. The kinetic parameters were determined using $10 \mu \mathrm{M}$ DddK reconstituted with $60 \mu \mathrm{M} \mathrm{Ni(II)} \mathrm{and} \mathrm{varying} \mathrm{concentrations} \mathrm{of} \mathrm{DMSP.}$

A

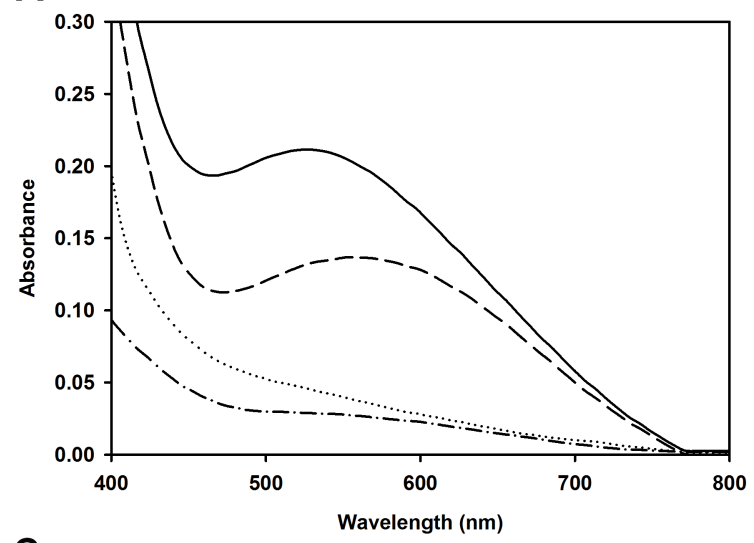

C

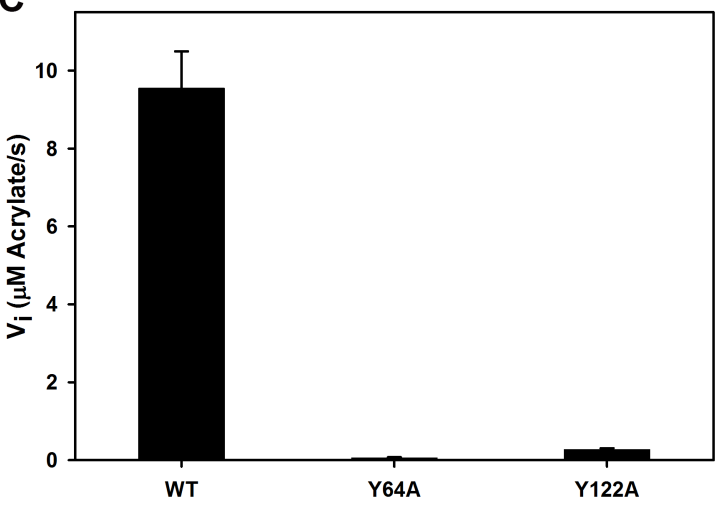

B

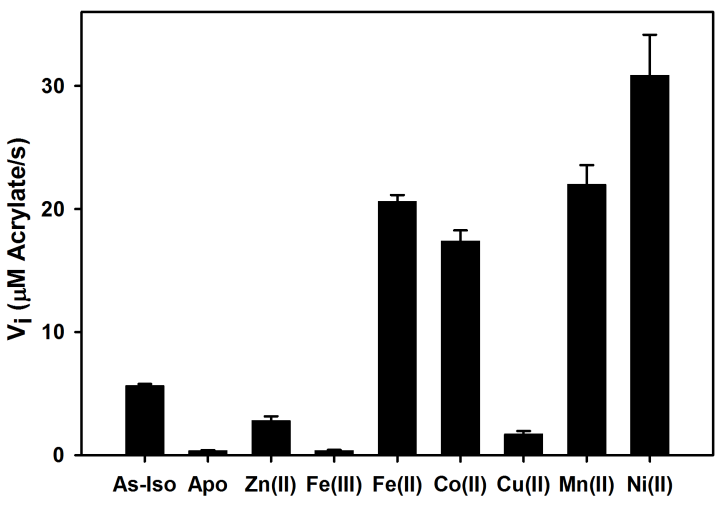

D

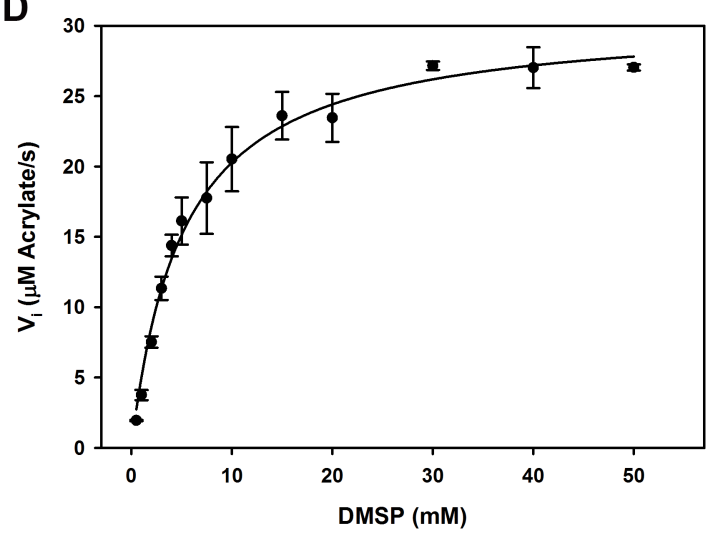


Metal ion dependence of DMSP cleavage by DddK. As-isolated DddK was active and the observed activity is mainly due to the presence of $\mathrm{Ni}(\mathrm{II}), \mathrm{Mn}(\mathrm{II})$, and some $\mathrm{Zn}$ (II) species (Figure 2B). Using the extinction coefficient for Fe(III)-tyrosinate $\left(1300 \mathrm{M}^{-1} \mathrm{~cm}^{-1}\right)^{32}$ and metal content results, we estimated that the as-isolated enzyme contains $\sim 45 \%$ of inactive species (Fe(III)-bound + apo-protein) and $55 \%$ active species. In order to understand catalytic efficiency of DddK with metal ions, an apo-form of the enzyme was used. The DMSP cleavage activity of DddK in the presence of various transition metal ions, such as, $\mathrm{Mn}(\mathrm{II}), \mathrm{Fe}(\mathrm{II}), \mathrm{Fe}(\mathrm{III}), \mathrm{Co}(\mathrm{II}), \mathrm{Ni}(\mathrm{II}), \mathrm{Cu}(\mathrm{II})$, and $\mathrm{Zn}(\mathrm{II})$ were examined by HPLC as described previously. ${ }^{17}$ DddK displayed maximum activity at $\mathrm{pH} 8.5$, which is consistent with the optimum alkaline growth environment of many marine bacteria (Figure S1B). ${ }^{34,35}$ The binding affinities of DddK for various metal ions were determined from enzymatic activity measurements using varying concentrations of metal ions at saturated DMSP concentration (Figure S2). All of the metal ions examined displayed positive cooperativity with Hill coefficients in the range of 3.6-5.1 and bind DddK with a $K_{d}$ in the range of 28-50 $\mu \mathrm{M}$ (Figure S2), indicating that the enzyme undergoes some conformational change in the presence of substrate/metal ions. Interestingly, metal binding to DddK was cooperative, whereas, kinetic data in the presence of varied DMSP concentration is not. The metal binding studies were also performed at a lower DMSP concentration below the measured $K_{m}(\sim 5 \mathrm{mM})$ and the results indicate similar cooperative behavior and $K_{d}$ values (data not shown). DddK being dimeric, perhaps metal binding at one site induces some subtle conformational change allowing a second metal ion to bind at another site (observed positive homotropic cooperativity), irrespective of the substrate concentration. The catalytic rate enhancement in the presence of various metal ions follows the order: $\mathrm{Ni}(\mathrm{II})>\mathrm{Mn}(\mathrm{II})>\mathrm{Fe}(\mathrm{II})>\mathrm{Co}(\mathrm{II})>$ $\mathrm{Zn}(\mathrm{II})>\mathrm{Cu}(\mathrm{II})>\mathrm{Fe}(\mathrm{III})$ (Figure 2B). 
For many metalloenzymes, the protein scaffold plays an important role in determining the ideal spatial organization of the metal binding residues, which typically confers optimum metal coordination geometry and contribute towards metal ion selectivity for optimal enzyme activity. ${ }^{36,37}$ Determining which metal ions are biologically available to DddK in the native organism is a very complex question. However, some hypotheses can be made by analysis of the environment in which the organism lives and the types of metal transporter proteins used by the organism. P. ubique strain HTCC1062 contains a streamlined genome of only about 1,300 genes and is found in low nutrient areas in the ocean. ${ }^{38,39}$ Of the metal ions examined, nickel is most abundant in the oceans, ${ }^{40,41}$ although local concentrations may vary across regions. ${ }^{42}$ A study of trace metal acquisition by marine bacteria has shown that SAR11 bacteria might only respond to a portion of the overall trace metals present in seawater. ${ }^{43}$ Indeed, SAR11 bacteria have significantly downsized metal dependent regulatory networks compared to the Roseobacter clade. ${ }^{43}$ While this study did not find any specific nickel transporter in the HTCC1062 genome, nickel transporter genes homologous to hupE and ureJ mostly co-localized with known transport proteins and are present in $45 \%$ of SAR11 genomes and $90 \%$ of Roseobacter genomes. ${ }^{43}$ HTCC1062 does however contain TolQ, a component of a larger complex that transports essential nutrients, such as nickel and iron, across the bacterial outer membrane. ${ }^{44}$ It is noteworthy that the majority of metal regulatory pathways characterized have been from non-marine organisms. Thus, it is probable that marine bacteria utilize metal transport systems that have not been discovered yet, e.g., MntX for Mn transport in SAR11 bacteria and some Vibrios. ${ }^{43,45}$ From an evolutionary perspective, it is sensible that a promiscuous metal dependent enzyme would evolve to utilize the most prevalent and capable metal cofactor.

In order to identify the catalytic roles of Tyr 122 and Tyr 64 (contributing to Fe(III)OTyr LMCT), the activity of DddK variants Y64A and Y122A were examined. Interestingly, 
the DddK variants had negligible DMSP cleavage activity in the absence and presence of the catalytically most active metal ion Ni(II), demonstrating that both Tyr 64 and Tyr 122 are necessary for metal binding and/or catalysis (Figure 2C).

The DMSP concentration dependence on DddK activity followed Michaelis-Menten kinetics at $\mathrm{pH} 8.5$ and room temperature. In the presence of $\mathrm{Ni}(\mathrm{II})$, DddK catalyzed the cleavage of DMSP with a $K_{m}$ for DMSP of $5.1 \pm 0.4 \mathrm{mM}$ and $k_{\text {cat }}$ of $3.1 \pm 0.1 \mathrm{~s}^{-1}$ (Figure 2D). This $K_{m}$ value is within the range reported for DMSP lyases $(0.4-22 \mathrm{mM})$ in the literature. ${ }^{11}$ The higher $K_{m}$ values reported for the DMSP lyases has been addressed before, but is largely believed to be related to the high intracellular concentrations of DMSP present in reported organisms. ${ }^{11}$ Such high DMSP concentrations are supported by the predicted DMSP transporter (OpuAC) being the sixth most abundant Pelagibacterales protein present in a Sargasso Sea metaproteome ${ }^{46}$ and likely occur because DMSP plays various roles in SAR11 bacteria as, e.g., an osmoprotectant or antioxidant. The overall catalytic efficiency $\left(k_{c a t} / K_{m}\right.$ of $\left.608 \pm 0.1 \mathrm{M}^{-1} \mathrm{~s}^{-1}\right)$ of $\mathrm{Ni}(\mathrm{II})$-DddK is comparable with that observed for DddW $\left(k_{c a t} / K_{m}\right.$ of 2.10 $\mathrm{x} 10^{3} \mathrm{M}^{-1} \mathrm{~s}^{-1}$, for Fe(II)-DddW), ${ }^{17}$ but 1000 times higher than DddQ $\left(k_{\text {cat }} / K_{m}\right.$ of $0.70 \pm 0.16 \mathrm{M}^{-}$ ${ }^{1} \mathrm{~s}^{-1}$, for Fe(II)-DddQ). ${ }^{19}$ Furthermore, the catalytic efficiency of Ni(II)-DddK is similar to that reported for the DMSP demethylation enzyme DmdA $\left(k_{c a t}\right.$ of $8.1 \mathrm{~s}^{-1}, K_{m}$ of $13.2 \mathrm{mM}$, and $k_{\text {cat }} / K_{m}$ of $614 \mathrm{M}^{-1} \mathrm{~s}^{-1}$ ) ${ }^{47}$, which is also from the same bacteria (i.e., P.ubique HTCC1062) as DddK.

Several models have been proposed for the existence of both the cleavage and demethylase pathways in DMSP degrading organisms., ${ }^{9}$ Sun et al., proposed that in Pelagibacter HTCC1062 the DMSP demethylation and cleavage pathways are controlled by the kinetics of the DmdA and DddK enzymes respectively. The demethylation pathway would be favored when intracellular levels of DMSP are low, and vice versa when levels are high, principally due to differences in their reported $K_{m}$ for DMSP $\left(K_{m}\right.$ of DmdA, $13.2 \mathrm{mM}$ 
and $82 \mathrm{mM}$ for DddK). ${ }^{9,47}$ However, our measured $K_{m}$ of Ni(II)-DddK (5 mM) for DMSP is lower than that determined previously for DddK $(82 \mathrm{mM})^{9}$ and that reported for DmdA (13.2 $\mathrm{mM}),{ }^{47}$ which we believe could be a result of differences in metal speciation, occupancy, or buffer system. Having a less preferred metal ion present in the active site as well as not having enough of the metal to occupy $100 \%$ of the enzyme molecules could affect the binding of DMSP to DddK. In addition, the use of a phosphate buffering system could be inhibiting DMSP binding. Our results show that enzyme metallation can make a sizable difference in comparing kinetic values and could potentially influence the contribution of the DMSP lysase and demethylation pathways in SAR11 bacteria accordingly.

Overall structure of DddK. DddK is the first cupin-fold containing DMSP lyase for which the structure of a highly active enzyme has been determined. The crystal structures of two different forms of DddK are determined, both obtained by crystallizing the as-isolated enzyme. The Ni-DddK structure determined at $2.2 \AA$, contains two molecules in the asymmetric unit with catalytic $\mathrm{Ni}(\mathrm{II})$ present at the active site along with a bound diacrylate (2-carboxyethyl acrylate) molecule (Figures 3A, 3B). Further investigation suggested that diacrylate is a dimerization product of acrylate and is a common contaminant found in acrylic acid. The mass spectrum of a sample from stock acrylic acid used in crystallization experiments showed a peak for diacrylate at $\mathrm{m} / \mathrm{z} 143.0356$, which accounted for $\sim 28 \%$ of the total sample (Figure S3). While Ni-DddK crystals were soaked with the product acrylate, the acrylate-bound species was not observed in the crystal and instead the relatively minor contaminant diacrylate was preferentially bound in the structure.

Figure 3. (A) Overall structure of $\mathrm{Ni}(\mathrm{II})$-DddK with the $\beta$-strands labeled as in Figure 1. Chain A is colored in green and chain B in blue. Nickel is shown as a green-colored sphere and diacrylate (cornflower blue) are shown as sticks. (B) Surface representation of Ni-DddK with chain $\mathrm{A}$ in green, chain $\mathrm{B}$ in blue, and interface residues in magenta. The metal binding residues His 56, His 58, Glu 62, His 93 and diacrylate (cornflower blue) are shown as sticks: carbon in protein backbone color, oxygen in red, and nitrogen in blue. (C) Detailed view of half of the symmetrical network of interactions between molecule A (green carbons) and molecule B (blue carbons) with hydrogen bonds indicated by black dashes (D) Anomalous 
A

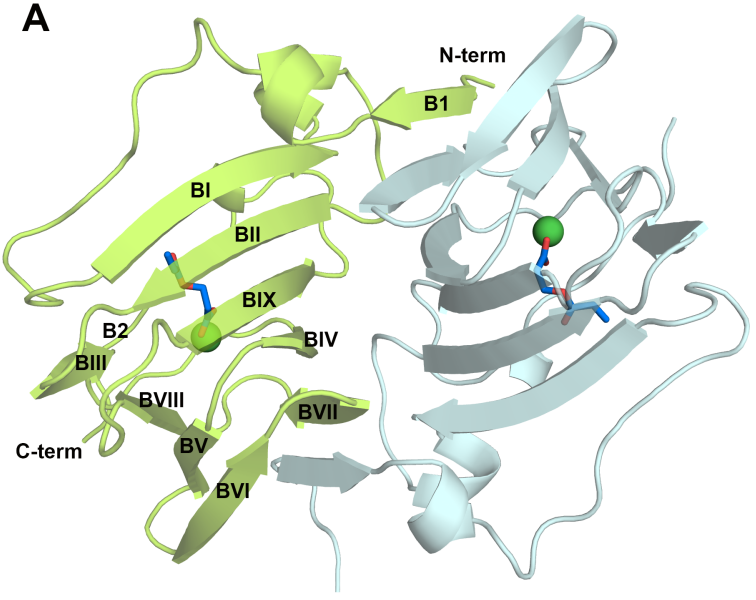

C

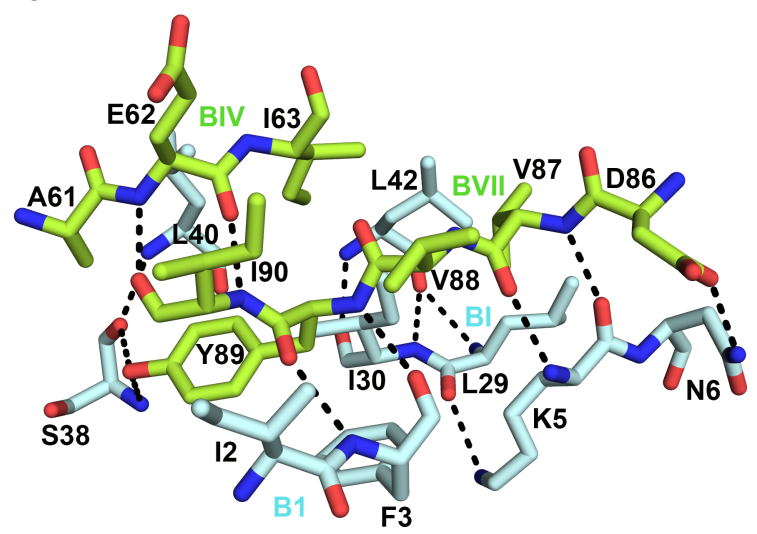

B

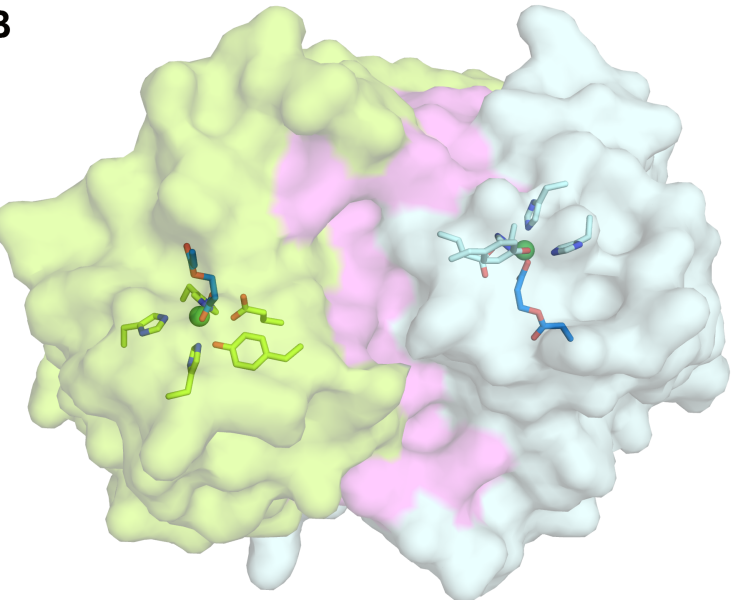

D

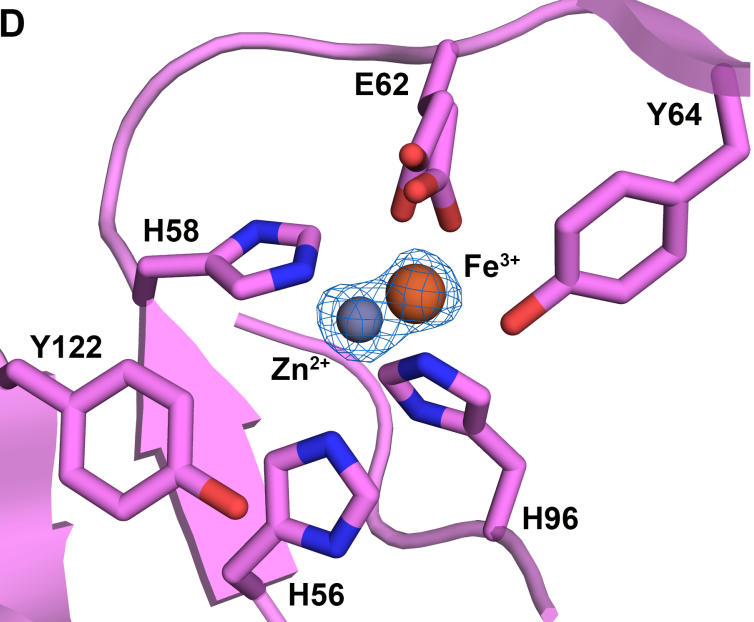

The as-isolated DddK protein contained only $7 \% \mathrm{Ni}$ based on ICP-OES analysis, however, nickel was found in the crystal at $100 \%$ occupancy. The presence of nickel was confirmed by the appearance of a Ni peak at $7.48 \mathrm{keV}$ in a fluorescence emission scan of the crystal used for data collection (Figure S4A). Furthermore, solution studies including sizeexclusion chromatography and dynamic light scattering of DddK predict a dimer $(\sim 28 \mathrm{kDa})$, which is also observed in the crystal structure. A large network of van der Waals and hydrogen bonding interactions are involved in stabilizing the dimer interface (Figure 3C). The dimer interface buries about $1560 \AA^{2}$ between both chains and contains 21 hydrogenbonding interactions. 
The second structure (FeZn-DddK) solved at $1.44 \AA$ resolution contains only one molecule in the asymmetric unit and has iron and zinc bound in the active site at different occupancies (0.6 Fe and $0.4 \mathrm{Zn}$ in the crystal), which is slightly different from that observed in ICP-OES analysis (Figure 3D). The fluorescence scans on the crystal showed clear signals for iron $(6.40 \mathrm{keV})$ and zinc $(8.64 \mathrm{keV})$ (Figure S4B). This is not surprising, given that metal analysis of as-isolated protein showed the presence of $40 \% \mathrm{Fe}$ and $20 \% \mathrm{Zn}$ bound to DddK. However, because of a large sampling error in crystallography, it is also possible to obtain a crystal with only one metal population (as observed in Ni-DddK structure) or mixed metal populations. Nonetheless anomalous density clearly shows the location of 2 distinct metal ion sites with partial occupancies (Figure 3D) and the as-isolated protein is slightly active due to zinc and nickel (Figure 2B).

DddK is composed of a single cupin domain that consists of two sides of anti-parallel $\beta$ sheets, with eight strands forming the DSBH fold (Figures 3A, 4A). The two chains in NiDddK are the same with a root mean square deviation (r.m.s.d.) of $0.19 \AA$ over $113 \mathrm{C}_{\alpha}$ atoms. The Ni-DddK and FeZn-DddK structures are also very similar with an r.m.s.d. of $0.31 \AA$ over $116 \mathrm{C}_{\alpha}$ atoms (Figure 4A). FeZn-DddK contains additional water molecules bound to the metal ions, however the substrate-binding pocket includes the N-terminal region from a neighboring symmetry related molecule. Both structures have missing densities for residues at the $\mathrm{N}$ - and $\mathrm{C}$-termini that were not included in the final model (see experimental procedures). In FeZn-DddK, there is a disruption in $\beta$ III from residues Gly 51 to His 56 compared to Ni-DddK, which causes $\beta$ III to be slightly shorter, but many of the side chains are quite similar (Figure 4A).

Figure 4. (A) Structural overlay of Ni-DddK (green) and FeZn-DddK (magenta) monomers. Highlighted differences for Ni-DddK are in orange, while differences for FeZn-DddK are in blue. Nickel is shown as a green-colored sphere and diacrylate (cornflower blue) are shown as sticks: carbon in backbone color, oxygen in red. Active sites of: (B) FeZn-DddK showing the $\mathrm{Fe}(\mathrm{III})$ coordination center. Ligands not involved in $\mathrm{Fe}(\mathrm{III})$ coordination were made transparent. (C) FeZn-DddK active site showing Zn(II) coordination center. Ligands not 
involved in $\mathrm{Zn}$ (II) coordination were made transparent. Note: $\mathrm{B}$ and $\mathrm{C}$ simply view the active site with one metal ion displayed at a time. (D) Diacrylate (a DMSP analog) is bound in a bidentate mode to $\mathrm{Ni}(\mathrm{II})$ in Ni-DddK. The $\mathrm{F}_{\mathrm{o}}-\mathrm{F}_{\mathrm{c}}$ omit map (green mesh) is contoured at $3 \sigma$ and a $2 \mathrm{~F}_{\mathrm{o}}-\mathrm{F}_{\mathrm{c}}$ composite omit map (gray mesh) is contoured at $1 \sigma$, both for diacrylate. (E) Cross-eyed stereo-view of DddK structures highlighting conformational changes involving Tyr 122 upon nickel and diacrylate binding. Active site comparison between Ni-DddK "closed" (green) and FeZn-DddK "open" (magenta) active sites. For B-E, protein residues and diacrylate (cornflower blue) are shown as sticks: carbon in backbone color, oxygen in red, and nitrogen in blue. Nickel (green), iron (rust), and zinc (gray) are shown as sphere. 
A

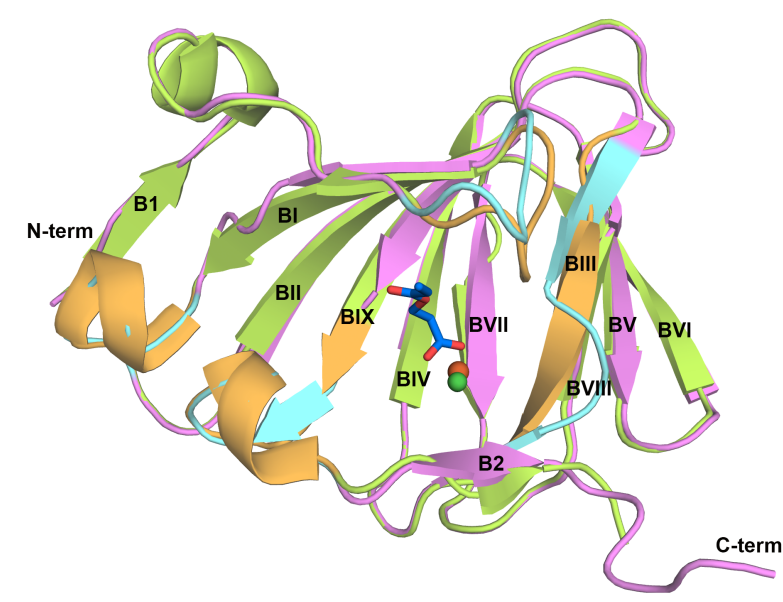

C

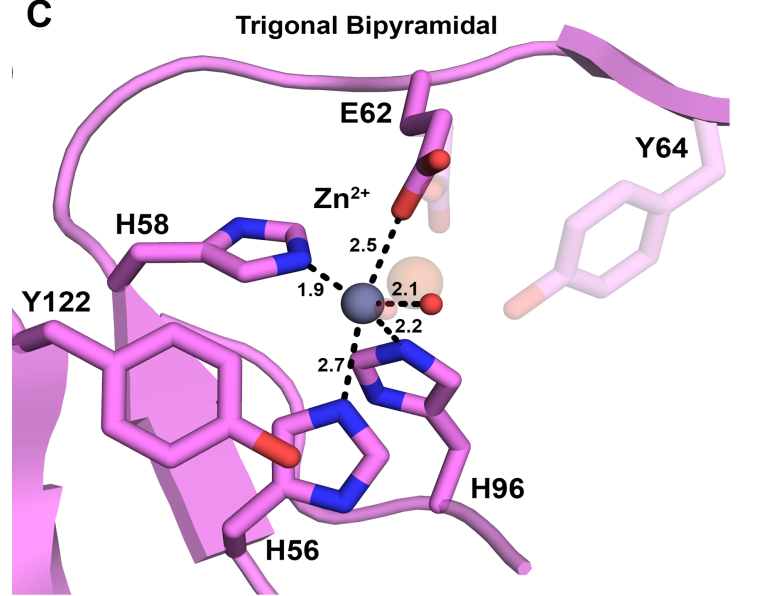

E

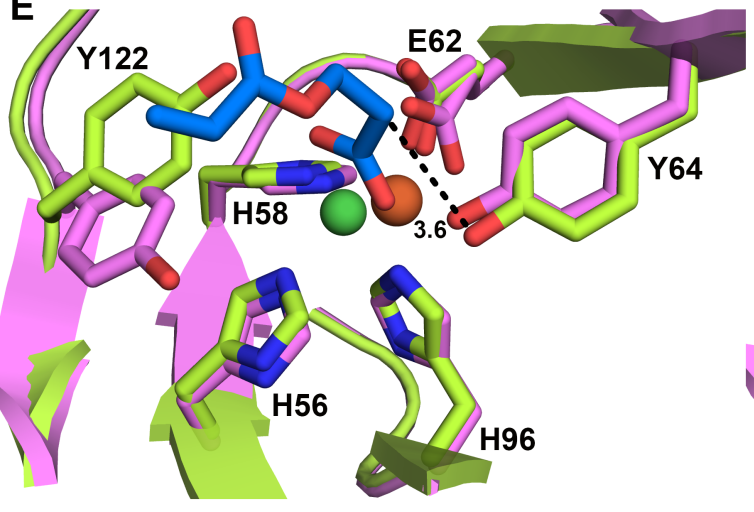

B

Octahedral

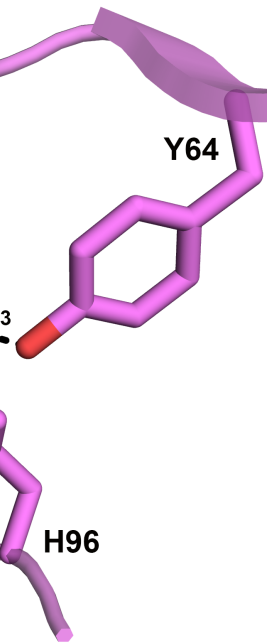

D
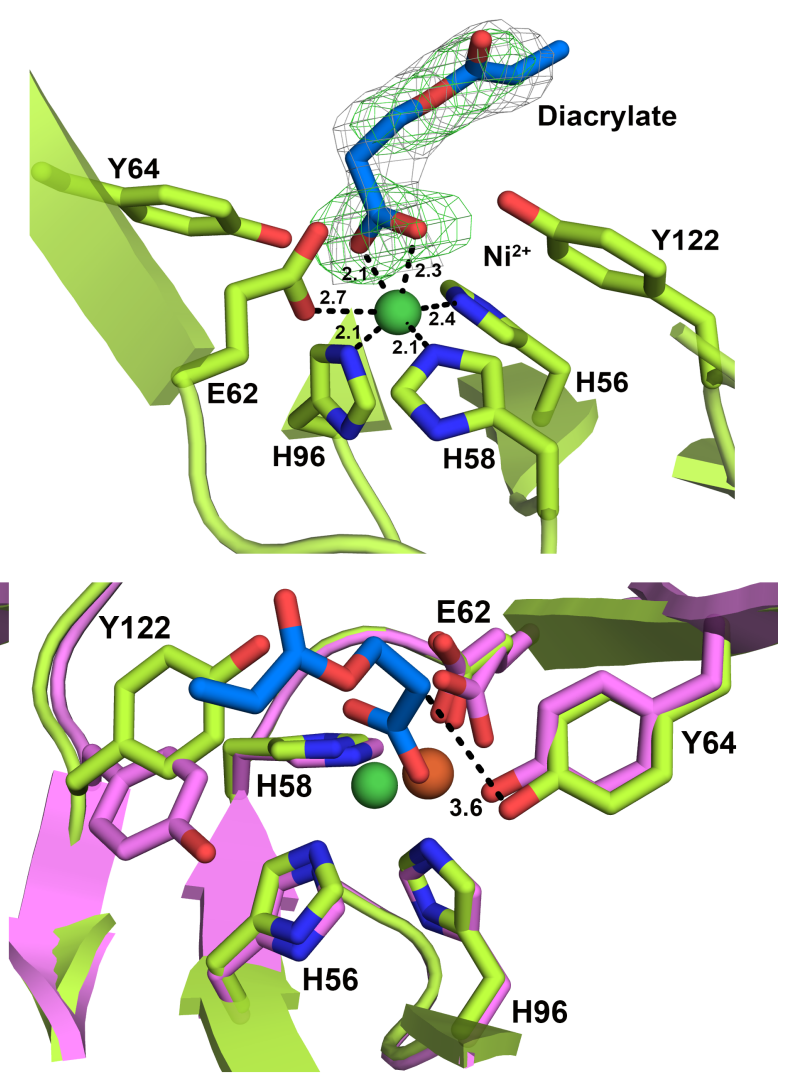

Metal coordination environments in the active sites of FeZn-DddK and Ni-DddK.

The active sites of the Ni-DddK and FeZn-DddK are distinct in terms of bound metal ions and their coordinated ligands (Figures 3D, 4). The coordination spheres around the metal ions in the active sites of the Ni-DddK and FeZn-DddK structures display important differences 
for DMSP lyase activity. In FeZn-DddK, anomalous density revealed clearly the positions of iron and zinc at the active site (Figure 3D). The distance between the iron and zinc atoms is too close $(1.3 \AA)$ for a possible dinuclear metal site. The exact locations of the metal ions were determined by interpretation of the solution UV-visible spectrum as well as chemical sense. On the basis of the Fe(III)-tyrosinate LMCT band, Fe(III) must be located in the metal position that is closer to Tyr 64 ligand (Figure 4B). In separate studies of metal environments present in proteins, iron was found to prefer both oxygen and nitrogen containing ligands, ${ }^{48}$ whereas zinc had a higher propensity for sulfur and nitrogen containing ligands. ${ }^{49,50}$ Due to the $\mathrm{Fe}(\mathrm{III})$-tyrosinate interaction and the preference of zinc for nitrogen over oxygen containing ligands, the zinc ion was modeled in the metal position closer to the three His residues (Figure 4C).

In the FeZn-DddK structure, the ligands coordinated to Fe(III) are His 58, Glu 62, Tyr 64, and His 96 at distances between 2.0-2.7 $\AA$, and a water molecule at 2.0 $\AA$. Glu 62 coordinates the $\mathrm{Fe}(\mathrm{III})$ center in a bidentate mode resulting in a 6-coordinate metal site with octahedral geometry (Figure 4B). The bidentate binding mode of a glutamate and the coordination of a tyrosinate to the iron center was observed in all ligand bound structures of Fe(III)-DddQ (Figure S5). ${ }^{19}$ Thus the FeZn-DddK structure reveals that Tyr 64 is responsible for the observed Fe(III)-tyrosinate LMCT transition. Furthermore, most of the equatorial ligands (Glu 62, His 96, and the water) have slightly smaller coordination distances (2.0-2.4 $\AA)$ than the axial ligands. The axial ligands, His 58 (2.7 $\AA)$ and Tyr 64 (2.3 $\AA)$, are slightly longer than the equatorial ligand coordination distances. The ligands coordinated to $\mathrm{Zn}$ (II) are His 56 , His 58, Glu 62, His 96, and a water molecule. Glu 62 is coordinated to the $\mathrm{Zn}(\mathrm{II})$ center in a monodentate binding fashion resulting in a 5-coordinate metal with trigonal bipyramidal geometry (Figure 4C). The monodentate binding of a glutamate was observed in the MES-bound structure of $\mathrm{Zn}(\mathrm{II})-\mathrm{DddQ} .{ }^{18}$ The equatorial ligands (His 58, His 96, and the 
water) have slightly smaller distances than the axial ligands (His 56 and Glu 62), which is expected for the trigonal bipyramidal geometry.

In the active Ni-DddK structure, the ligands coordinated to the Ni(II) are His 56, His 58 , Glu 62 , His 96 , and the carboxylate group of diacrylate, which are all at distances within 2.1-2.7 $\AA$ from the nickel center. Glu 62 coordinates in a monodentate fashion while the diacrylate is bound in a bidentate binding mode, resulting in a 6-coordinate metal center with octahedral geometry (Figure 4D). The binding of three histidines to the metal center along with a monodentate glutamate has not been observed previously with any reported DddQ structures. $^{18,19}$ In the Ni-DddK structure, the equatorial ligands (His 58, His 96, and diacrylate) had slightly shorter metal coordination distances than the axial ligands (His 56 and Glu 62), similar to that observed for the Fe(III) site in FeZn-DddK structure. A superposition of both structures shows that nickel occupies the same position as zinc (Figure 4E). The presence of diacrylate in the Ni-DddK structure does not allow Tyr 64 to bind to the $\mathrm{Ni}(\mathrm{II})$ center. This structure of the catalytically competent diacrylate bound Ni-DddK mimics the substrate bound form of the enzyme. Since Ni(II) has the maximum activity among all metal ions examined, therefore the preferred metal coordination geometry in presence of substrate for optimal DMSP lyase activity is octahedral.

Intramolecular interactions at DddK active site. The active site regions of both FeZnDddK and Ni-DddK are stabilized by a number of hydrogen bonds and hydrophobic interactions and most of these interactions are mediated by highly conserved residues such as His 58, Trp 110, and Tyr 21. In Ni-DddK two water molecules are located 5.5 and $5.2 \AA$ away from the nickel center and both interact with the side chain of His 58 (Figure 5A). One of these waters (W1) interacts with the backbone carbonyl oxygens of Pro 60, Ile 90, and Ala 91. The other water (W2) interacts with the His 58 backbone nitrogen and the backbone 
oxygen and nitrogen atoms of Ala 94. In addition, two other water molecules (W3 and W4) are within hydrogen-bonding distance from each other and interact with other residues. W3 interacts with the hydroxyl group of Ser 41 and the side chain of Trp 110, while W4 forms three hydrogen bonds. W4 is hydrogen bonded to Asp 31 carboxylate and interacts with the backbone carbonyls of Ile 30 and Leu 42. The diacrylate molecule is anchored in the active site through a hydrogen bond with Tyr $122(2.7 \AA)$ and is in close contact with Tyr $64(2.3$ $\AA$ ), with the side chain of Tyr 122 remaining in the protonated state. In addition, Tyr 64 is within hydrogen bonding distance to the metal coordinated His 96 (3.5 $\AA$ ).

Figure 5. (A) Contact map of active site residues of diacrylate-bound Ni-DddK between protein, ligand, and water are indicated by black dashes with corresponding distances. (B) Hydrogen bonding interactions for FeZn-DddK. The hydrogen bonding interactions between active site residues and water are shown in black dashes with corresponding distances. For A and $\mathrm{B}$, protein residues and diacrylate (cornflower blue) are shown as sticks: carbon in backbone color, oxygen in red, and nitrogen in blue. Nickel (green), iron (rust), zinc (gray), and water molecules (cyan) are shown as spheres.

A

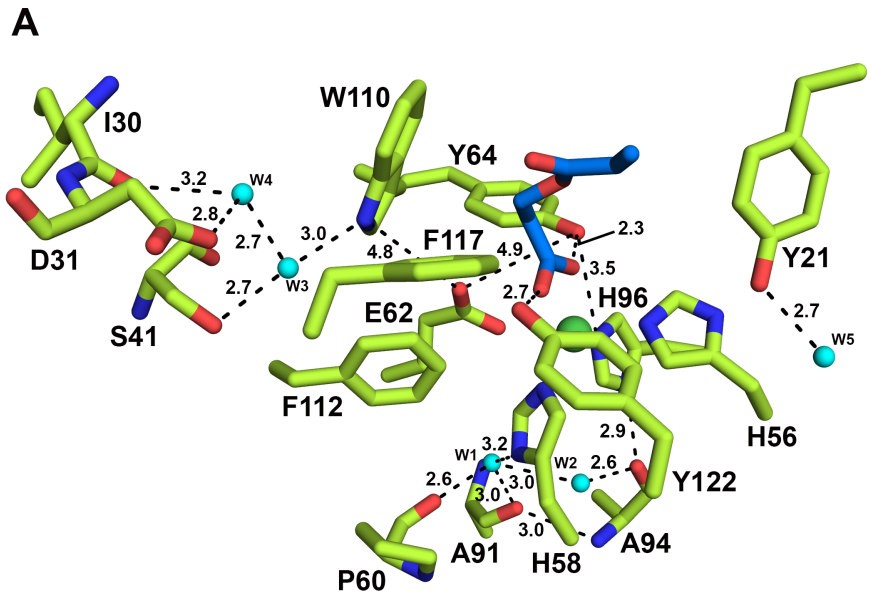

B

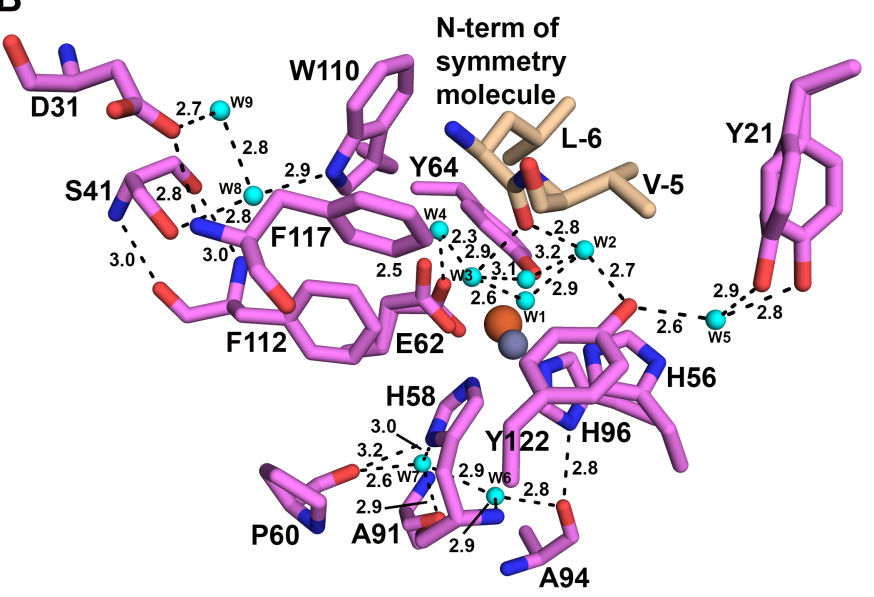

The FeZn-DddK structure has highly ordered water molecules in the active site. The absence of any bound ligand creates an extensive hydrogen-bonding network between the water molecules and the enzyme. Of particular interest are waters W2, W3, and W4. These waters are located in the diacrylate-binding region and interact with $\mathrm{W} 1$ that coordinates the metal ions (Figure 5B). The residues that are involved in hydrogen bonding interactions include His 56, His 58, Tyr 64, Tyr 122, Tyr 21, and Trp 110. 
Interestingly, Tyr 64 and Tyr 122 are involved in a hydrogen-bonding network spanning from the active site to the protein surface in both FeZn-DddK and Ni-DddK structures. Substitution of these Tyr residues leads to complete loss of DddK activity, thereby confirming that these Tyr residues are important for substrate positioning and catalysis. Furthermore, the deprotonated forms of these tyrosines could play an important role in proton abstraction when the substrate and appropriate metal ion are bound in the active site of DddK.

Ligand binding and conformational changes. Although the Ni-DddK and FeZn-DddK structures are similar, there are some differences worth noting. In diacrylate-bound Ni-DddK, the loop consisting of residues Thr 18-Gly 23, immediately before $\beta$-strand I (BSI) undergoes $2.5 \AA$ movement to accommodate diacrylate, which results in a slightly more closed active site compared to FeZn-DddK (Figures 4A and 4E). Tyr 21 in this region has different orientations between the structures and could be involved in substrate binding or release (Figure 5). In addition, Tyr 122 occupies two different positions in both DddK structures. Tyr 122 swings towards diacrylate in Ni-DddK by about $50^{\circ}$ to help coordinate the carboxylate (Figures 4E, 5). Although these movements could be related to differences in crystal packing, molecular dynamics simulation on DddQ revealed 2 regions that undergo similar movement to make the active site more "closed" and "open". ${ }^{18}$ While much of the active site is buried from solvent, analysis of the electrostatic potential surface of DddK around the substratebinding pocket shows a small channel available for DMS to exit the active site after catalysis (Figure 6). In the absence of any substrate or product, FeZn-DddK has one water molecule bound to each metal, located at 2.0 and $2.1 \AA$ away from the iron and zinc centers respectively (Figure 5B). Structural overlay shows that nickel occupies the same position as zinc (Figure 4E), which indicates the possibility of a shift in metal ion position in the 

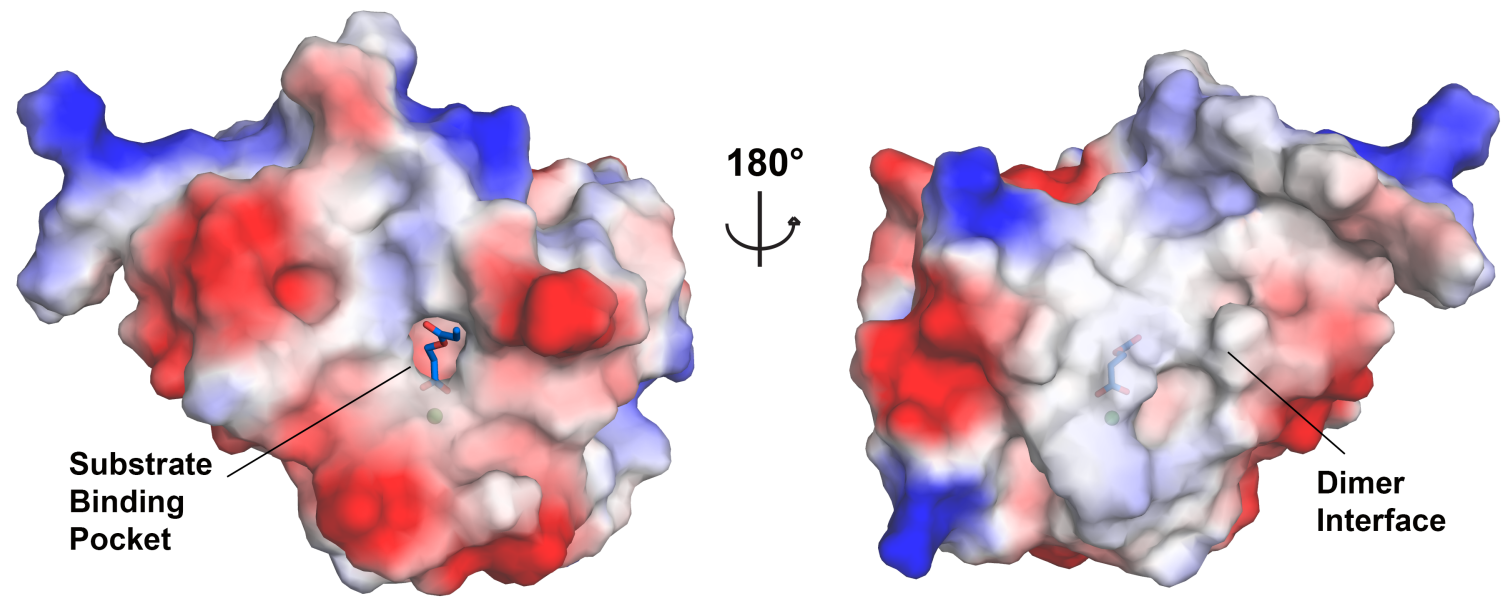

Mechanism of DMSP cleavage by DddK. On the basis of biochemical and structural studies it is established that the DMSP lyases produce acrylate and DMS via a $\beta$-elimination mechanism. ${ }^{17-19}$ However, it is yet to be determined how different metal cofactors together with the protein residues influence substrate binding and initiate catalysis. A $\mathrm{Zn}$ (II)dependent mechanism for DMSP cleavage by DddQ has been proposed, however, concerns regarding some of the findings are discussed in Alcolombri et al. ${ }^{51}$ The DMSP and acrylate bound structures of Fe(III)-DddQ nicely illustrated conformational changes in key residues upon DMSP binding and identified the tyrosine residue initiating catalysis. ${ }^{19}$ The results presented here with active $\mathrm{Ni}(\mathrm{II})$-bound DddK have revealed important catalytic residues and the impact of the nickel coordination environment for DMSP cleavage activity of DddK.

The FeZn-DddK structure together with absorption spectral studies with wild type DddK and variants clearly illustrate that Tyr 64 participates in coordinating with Fe(III) (Figure 2A). The UV-visible spectrum of apo-DddK in presence of $\mathrm{Ni}$ (II) shows weak absorption bands at $375 \mathrm{~nm}\left(272 \mathrm{M}^{-1} \mathrm{~cm}^{-1}\right), 610 \mathrm{~nm}\left(72 \mathrm{M}^{-1} \mathrm{~cm}^{-1}\right), 945 \mathrm{~nm}\left(102 \mathrm{M}^{-1} \mathrm{~cm}^{-1}\right)$, 
and $1020 \mathrm{~nm}\left(105 \mathrm{M}^{-1} \mathrm{~cm}^{-1}\right)$, indicating the formation of a Ni(II) complex with an octahedral coordination (Figure S6). ${ }^{52}$ A shoulder near $300 \mathrm{~nm}\left(\sim 860 \mathrm{M}^{-1} \mathrm{~cm}^{-1}\right)$ was observed that can be attributed to tyrosinate-Ni(II) interaction, given a $\mathrm{Ni}(\mathrm{II})$-phenolate chromophore has been shown to exhibit bands between $200-400 \mathrm{~nm}^{53}$ and a Ni(II)-thiolate species has an absorption feature at $315 \mathrm{~nm} .{ }^{54,55}$ In contrast, intense LMCT transitions due to Ni(II)-phenolate that appear between $400-600 \mathrm{~nm}\left(\varepsilon>900 \mathrm{M}^{-1} \mathrm{~cm}^{-1}\right)^{56,57}$ were not present. Interestingly, in the diacrylate bound Ni(II)-DddK structure, Tyr 64 does not participate as a metal ligand (Figure 4D). Our hypothesis is that in the resting state, in absence of substrate Tyr 64 is deprotonated and coordinates to the $\mathrm{Ni}(\mathrm{II})$ center, as observed in the iron-bound DddK structure (Figures 4B, 7).

Figure 7. Proposed reaction mechanism of DMSP cleavage catalyzed by DddK. In the absence of substrate (I), a water molecule is bound to the Ni(II) center, while Tyr 64 remains ligated to nickel. When substrate approaches, Tyr 64 and the water molecule are displaced and DMSP coordinates nickel in a bidentate fashion through its carboxylate end (II). The tyrosine resides (Tyr 64 and Tyr 122) anchor DMSP and orient it ready for catalysis. This positioning of DMSP brings Tyr 64 in the proximity of $\mathrm{C} 2$ hydrogen for proton abstraction (III). Subsequent cleavage of C3 carbon-sulfur bond produces DMS and acrylate (IV). The acrylate is shown to bind to metal center in a bidentate mode, however a monodentate binding of acrylate is also possible as observed in DddQ. ${ }^{19}$ 

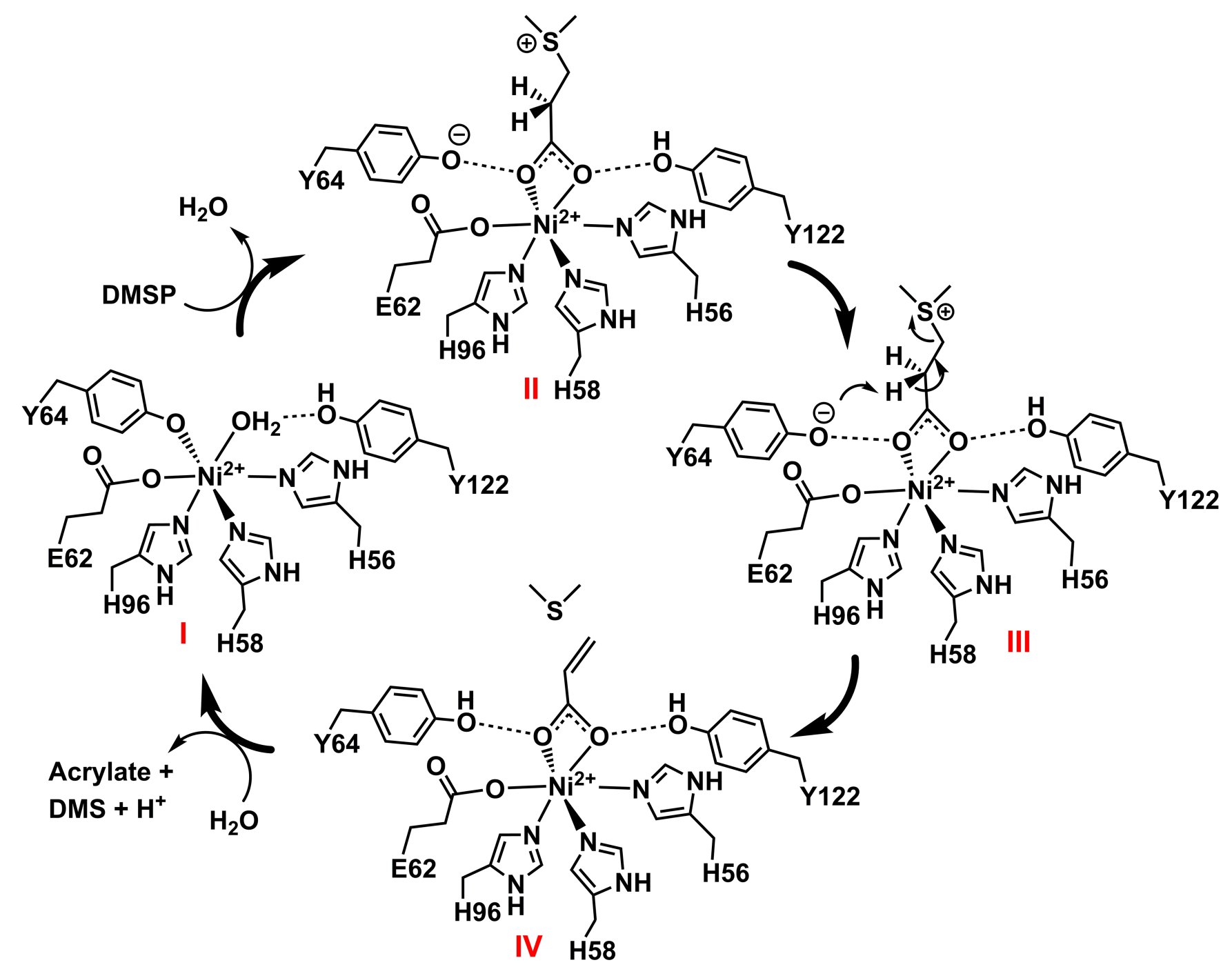

II H58

In the presence of the substrate, Tyr 64 moves to a catalytic transition state causing it to no longer coordinate the $\mathrm{Ni}$ (II) center. In the catalytic transition state of Ni-DddK, both Tyr 64 and Tyr 122 help anchor the carboxylate end of diacrylate to the Ni(II) center. Because of the metal coordination and close proximity of Tyr 64 to the electrostatically positive nickel center $(3.9 \AA)$, the pKa is most likely lowered producing the deprotonated form ready to initiate catalysis. This type of "tyrosine switch" mechanism has been previously described for zinc containing serralysins and astacins. ${ }^{58,59}$ Alternatively, it is possible that the activation of Tyr 64 promoting it to the anionic state could be achieved by neighboring His 96 (Figure 5A). The possible involvement of a histidine in aiding the deprotonated state of a nearby tyrosine has been proposed on the basis of a DMSP-bound structure of Fe(III)-DddQ. ${ }^{19}$ Thus in the substrate bound transition state structure of Ni-DddK, the negatively charged Tyr 64 
residue is well-positioned for proton abstraction and is $3.6 \AA$ away from what would be the $\mathrm{C}_{\alpha}$ of DMSP (Figure 4E). Tyr 122 swings $5.3 \AA$ from FeZn-DddK to its position in Ni-DddK (Figure 4E), which is necessary for DddK activity. Tyr 122 is likely not involved in proton abstraction but is probably important for transition state stabilization.

In addition to revealing that Tyr 64 is responsible for the $\mathrm{C}_{\alpha}$ proton abstraction from DMSP, we have established that a distorted octahedral metal environment is preferred for DddK activity. The metal ions $\mathrm{Ni}(\mathrm{II}), \mathrm{Mn}(\mathrm{II}), \mathrm{Co}(\mathrm{II})$, and $\mathrm{Fe}(\mathrm{II})$ can all form octahedral geometries and display DMSP lyase activity. Activity decreases considerably for $\mathrm{Zn}(\mathrm{II})$ and $\mathrm{Cu}(\mathrm{II})$ as both these ions prefer geometries other than octahedral in protein environments. While octahedral geometry is observed for Fe(III), the iron is bound in a slightly shifted position from $\mathrm{Ni}(\mathrm{II})$. More importantly $\mathrm{Fe}(\mathrm{III})$ is ligated to Tyr 64 in the FeZn-DddK structure in the absence of substrate and presumably in the presence of substrate, thus making it inaccessible for $\mathrm{C}_{\alpha}$ proton abstraction, which is supported by the negligible activity observed in presence of Fe(III) (Figure 2B).

On the basis of the crystal structures and the biochemical data, a mechanism of DddK catalyzed $\beta$-elimination of DMSP is proposed (Figure 7). The active Ni(II) center is coordinated by 3His-1-Glu-1-Tyr (H56, H58, E62, Y64, and H96) and the remaining open coordination site is occupied by a water molecule in the resting state (I, Figure 7) of the enzyme. While the structure of Ni-DddK with bound water is not yet available, one can visualize the occurrence of complex (I) on the basis of Fe(III)-DddK active site, which contains a bound water in the absence of substrate (Figures $4 \mathrm{~B}, \mathrm{C}$ ). When DMSP enters the active site, the metal bound water and Tyr 64 are displaced and the substrate binds in a bidentate mode (II, Figure 7) as observed in the diacrylate bound Ni-DddK structure. However, DMSP can also bind to the metal center in a monodentate fashion as seen in the previously published DMSP-bound Fe(III)-DddQ structure. ${ }^{19}$ Both monodentate and 


\section{Conclusions}

The studies presented here provide significant insights into the understanding of DddK-catalyzed DMSP cleavage by ubiquitous SAR11 marine bacteria. Given the prevalence of SAR11 bacteria in the oceans and that many strains contain DddK, understanding of the mechanism of DddK catalyzed DMSP cleavage is an important piece of the global sulfur cycle puzzle. Indeed, our findings demonstrate that when reconstituted with certain metal ions such as $\mathrm{Ni}(\mathrm{II})$, DddK is as efficient as DmdA. It is possible that this is the reason why lab cultures of Pelagibacterales strain HTCC1062 shunted $\sim 60 \%$ of DMSP via the DddK DMSP lyase pathway. ${ }^{9}$ The Ni-bound structure presented here resembles the catalytic state of DddK. The results support that Tyr 64 in DddK is responsible for the $\beta$ elimination leading to the production of acrylate and DMS. This work is the first to offer an explanation for the varying metal dependence observed for DMSP lyase activity. While determining the metal speciation of DddK in the native organism would be of clear interest, ${ }^{60}$ due to issues such as sluggish growth of SAR 11 and very low yielding cultures, it is not experimentally feasible. Although practically problematic, studies of local metal 
environments found in native organism surroundings, as well as a more complete understanding of metal transport and homeostasis for specific organisms, are necessary to develop truly valuable climate models for DMSP cleavage and other processes involving metalloenzymes.

Acknowledgements. We would like to thank Stephen Giovannoni and Rob Green for useful discussions and Nick Le Brun for reviewing the manuscript. M. D. was supported by start-up funds from the University of Iowa College of Liberal Arts and Sciences and funding from the National Science Foundation (CLP 1506181). Funding from the Natural Environmental Research Council (NE/J01138X, NE/M004449 and NE/N002385) supported work in J.D.T.'s laboratory. X-ray data were collected at the Advanced Light Source (4.2.2) and Advanced Photon Source (19-ID-D). Support for the synchrotron sources is provided by the U.S. Department of Energy.

Data deposition. Crystallography, atomic coordinates, and structure factors of have been deposited in the Protein Data Bank, www.pdb.org (PDB ID codes 5TFZ and 5TGO).

Supporting Information. Metal content and optimal pH of DddK (Figure S1); Metal binding affinities of DddK (Figure S2); X-ray fluorescence emission spectra (Figure S3); Structural comparison of active sites of nickel-DddK and iron-DddQ (Figure S4); UV-visible spectrum of Ni(II)-bound DddK (Figure S5). This material is available free of charge via the Internet at http://pubs.acs.org.

\section{AUTHOR INFORMATION}

Corresponding Author.* E-mail: mishtu-dey@uiowa.edu 
ORCID. Mishtu Dey: 0000-0003-4763-2921

\section{References}

[1] Simo, R. (2001) Production of atmospheric sulfur by oceanic plankton: biogeochemical, ecological and evolutionary links, Trends Ecol. Evol. 16, 287-294.

[2] Kiene, R. P., Linn, L. J., Gonzalez, J., Moran, M. A., and Bruton, J. A. (1999) Dimethylsulfoniopropionate and methanethiol are important precursors of methionine and protein-sulfur in marine bacterioplankton, Appl. Environ. Microbiol. 65, 4549-4558.

[3] Kiene, R. P., Linn, L. J., and Bruton, J. A. (2000) New and important roles for DMSP in marine microbial communities, J. Sea Res. 43, 209-224.

[4] Curson, A. R., Todd, J. D., Sullivan, M. J., and Johnston, A. W. (2011) Catabolism of dimethylsulphoniopropionate: microorganisms, enzymes and genes, Nat. Rev. Microbiol. 9, 849-859.

[5] Dickschat, J. S., Rabe, P., and Citron, C. A. (2015) The chemical biology of dimethylsulfoniopropionate, Org. Biomol. Chem. 13, 1954-1968.

[6] Moran, M. A., Reisch, C. R., Kiene, R. P., and Whitman, W. B. (2012) Genomic insights into bacterial DMSP transformations, Ann. Rev. Mar. Sci. 4, 523-542.

[7] Reisch, C. R., Moran, M. A., and Whitman, W. B. (2011) Bacterial Catabolism of Dimethylsulfoniopropionate (DMSP), Front. Microbiol. 2, 1-12.

[8] Kettle, A. J., and Andreae, M. O. (2000) Flux of dimethylsulfide from the oceans: A comparison of updated data sets and flux models, J. Geophys. Res.: Atmospheres 105, 2679326808.

[9] Sun, J., Todd, J. D., Thrash, J. C., Qian, Y., Qian, M. C., Temperton, B., Guo, J., Fowler, E. K., Aldrich, J. T., Nicora, C. D., Lipton, M. S., Smith, R. D., De Leenheer, P., Payne, S. H., Johnston, A. W., Davie-Martin, C. L., Halsey, K. H., and Giovannoni, S. J. (2016) The abundant marine bacterium Pelagibacter simultaneously catabolizes dimethylsulfoniopropionate to the gases dimethyl sulfide and methanethiol, Nat Microbiol 1, $1-5$.

[10] Alcolombri, U., Ben-Dor, S., Feldmesser, E., Levin, Y., Tawfik, D. S., and Vardi, A. (2015) MARINE SULFUR CYCLE. Identification of the algal dimethyl sulfide-releasing enzyme: A missing link in the marine sulfur cycle, Science 348, 1466-1469.

[11] Johnston, A. W., Green, R. T., and Todd, J. D. (2016) Enzymatic breakage of dimethylsulfoniopropionate-a signature molecule for life at sea, Curr. Opin. Chem. Biol. 31, 58-65.

[12] Dunwell, J. M., Culham, A., Carter, C. E., Sosa-Aguirre, C. R., and Goodenough, P. W. (2001) Evolution of functional diversity in the cupin superfamily, Trends Biochem. Sci. 26, 740-746.

[13] Dunwell, J. M., Purvis, A., and Khuri, S. (2004) Cupins: the most functionally diverse protein superfamily?, Phytochemistry 65, 7-17.

[14] Hajnal, I., Lyskowski, A., Hanefeld, U., Gruber, K., Schwab, H., and Steiner, K. (2013) Biochemical and structural characterization of a novel bacterial manganese-dependent hydroxynitrile lyase, FEBS J. 280, 5815-5828.

[15] Chavez, F. A., Banerjee, A., and Sljivic, B. (2011) Modeling the Metal Binding Site in Cupin Proteins, Vol. InTech, Rijeka, Croatia.

[16] Khuri, S., Bakker, F. T., and Dunwell, J. M. (2001) Phylogeny, function, and evolution of the cupins, a structurally conserved, functionally diverse superfamily of proteins, Mol. Biol. Evol. 18, 593-605. 
[17] Brummett, A. E., Schnicker, N. J., Crider, A., Todd, J. D., and Dey, M. (2015) Biochemical, Kinetic, and Spectroscopic Characterization of Ruegeria pomeroyi DddW--A Mononuclear Iron-Dependent DMSP Lyase, PLoS One 10, e0127288.

[18] Li, C. Y., Wei, T. D., Zhang, S. H., Chen, X. L., Gao, X., Wang, P., Xie, B. B., Su, H. N., Qin, Q. L., Zhang, X. Y., Yu, J., Zhang, H. H., Zhou, B. C., Yang, G. P., and Zhang, Y. Z. (2014) Molecular insight into bacterial cleavage of oceanic dimethylsulfoniopropionate into dimethyl sulfide, Proc. Natl. Acad. Sci. U. S. A. 111, 1026-1031.

[19] Brummett, A. E., and Dey, M. (2016) New Mechanistic Insight from Substrate- and Product-Bound Structures of the Metal-Dependent Dimethylsulfoniopropionate Lyase DddQ, Biochemistry 55, 6162-6174.

[20] Morris, R. M., Rappe, M. S., Connon, S. A., Vergin, K. L., Siebold, W. A., Carlson, C. A., and Giovannoni, S. J. (2002) SAR11 clade dominates ocean surface bacterioplankton communities, Nature 420, 806-810.

[21] Schnicker, N. J., and Dey, M. (2016) Bacillus anthracis Prolyl 4-Hydroxylase Modifies Collagen-like Substrates in Asymmetric Patterns, J. Biol. Chem. 291, 13360-13374.

[22] Knauer, S. H., Hartl-Spiegelhauer, O., Schwarzinger, S., Hanzelmann, P., and Dobbek, H. (2012) The Fe(II)/alpha-ketoglutarate-dependent taurine dioxygenases from Pseudomonas putida and Escherichia coli are tetramers, FEBS J. 279, 816-831.

[23] Schnicker, N. J., and Dey, M. (2016) Structural analysis of cofactor binding for a prolyl 4-hydroxylase from the pathogenic bacterium Bacillus anthracis, Acta Crystallogr D Struct Biol 72, 675-681.

[24] Kabsch, W. (2010) Xds, Acta Crystallogr. D Biol. Crystallogr. 66, 125-132.

[25] Evans, P. R., and Murshudov, G. N. (2013) How good are my data and what is the resolution?, Acta Crystallogr. D Biol. Crystallogr. 69, 1204-1214.

[26] McCoy, A. J., Grosse-Kunstleve, R. W., Adams, P. D., Winn, M. D., Storoni, L. C., and Read, R. J. (2007) Phaser crystallographic software, J. Appl. Crystallogr. 40, 658-674.

[27] Afonine, P. V., Grosse-Kunstleve, R. W., Echols, N., Headd, J. J., Moriarty, N. W., Mustyakimov, M., Terwilliger, T. C., Urzhumtsev, A., Zwart, P. H., and Adams, P. D. (2012) Towards automated crystallographic structure refinement with phenix.refine, Acta Crystallogr. D Biol. Crystallogr. 68, 352-367.

[28] Morin, A., Eisenbraun, B., Key, J., Sanschagrin, P. C., Timony, M. A., Ottaviano, M., and Sliz, P. (2013) Collaboration gets the most out of software, Elife 2, e01456.

[29] Berman, H., Henrick, K., and Nakamura, H. (2003) Announcing the worldwide Protein Data Bank, Nat. Struct. Biol. 10, 980.

[30] Chen, V. B., Arendall, W. B., 3rd, Headd, J. J., Keedy, D. A., Immormino, R. M., Kapral, G. J., Murray, L. W., Richardson, J. S., and Richardson, D. C. (2010) MolProbity: all-atom structure validation for macromolecular crystallography, Acta Crystallogr. D Biol. Crystallogr. 66, 12-21.

[31] Zheng, H., Chordia, M. D., Cooper, D. R., Chruszcz, M., Muller, P., Sheldrick, G. M., and Minor, W. (2014) Validation of metal-binding sites in macromolecular structures with the CheckMyMetal web server, Nat. Protoc. 9, 156-170.

[32] Huo, L., Fielding, A. J., Chen, Y., Li, T., Iwaki, H., Hosler, J. P., Chen, L., Hasegawa, Y., Que, L., Jr., and Liu, A. (2012) Evidence for a dual role of an active site histidine in alpha-amino-beta-carboxymuconate-epsilon-semialdehyde decarboxylase, Biochemistry 51, 5811-5821.

[33] Skrzypczak-Jankun, E., Bross, R. A., Carroll, R. T., Dunham, W. R., and Funk, M. O., Jr. (2001) Three-dimensional structure of a purple lipoxygenase, J. Am. Chem. Soc. 123, 10814-10820.

[34] Padan, E., Bibi, E., Ito, M., and Krulwich, T. A. (2005) Alkaline pH homeostasis in bacteria: new insights, Biochim. Biophys. Acta 1717, 67-88. 
[35] Krause, E., Wichels, A., Gimenez, L., Lunau, M., Schilhabel, M. B., and Gerdts, G. (2012) Small changes in $\mathrm{pH}$ have direct effects on marine bacterial community composition: a microcosm approach, PLoS One 7, e47035.

[36] Pennella, M. A., Shokes, J. E., Cosper, N. J., Scott, R. A., and Giedroc, D. P. (2003) Structural elements of metal selectivity in metal sensor proteins, Proc. Natl. Acad. Sci. U. S. A. 100, 3713-3718.

[37] Dudev, T., and Lim, C. (2014) Competition among metal ions for protein binding sites: determinants of metal ion selectivity in proteins, Chem. Rev. 114, 538-556.

[38] Giovannoni, S. J. (2017) SAR11 Bacteria: The Most Abundant Plankton in the Oceans, Ann. Rev. Mar. Sci. 9, 12.11-12.25.

[39] Carini, P., Steindler, L., Beszteri, S., and Giovannoni, S. J. (2013) Nutrient requirements for growth of the extreme oligotroph 'Candidatus Pelagibacter ubique' HTCC 1062 on a defined medium, ISME J 7, 592-602.

[40] Vraspir, J. M., and Butler, A. (2009) Chemistry of marine ligands and siderophores, Ann. Rev. Mar. Sci. 1, 43-63.

[41] Bruland, K. W., and Lohan, M. C. (2014) Controls of Trace Metals in Seawater, In Treatise on Geochemistry (Second Edition) (Turekian, K. K., Ed.), pp 19-51, Elsevier, Oxford.

[42] Cox, P. A. (1989) The elements : their origin, abundance, and distribution, Oxford University Press, Oxford, England.

[43] Hogle, S. L., Thrash, J. C., Dupont, C. L., and Barbeau, K. A. (2016) Trace Metal Acquisition by Marine Heterotrophic Bacterioplankton with Contrasting Trophic Strategies, Appl. Environ. Microbiol. 82, 1613-1624.

[44] Morris, R. M., Nunn, B. L., Frazar, C., Goodlett, D. R., Ting, Y. S., and Rocap, G. (2010) Comparative metaproteomics reveals ocean-scale shifts in microbial nutrient utilization and energy transduction, ISME J 4, 673-685.

[45] Green, R. T., Todd, J. D., and Johnston, A. W. (2013) Manganese uptake in marine bacteria; the novel MntX transporter is widespread in Roseobacters, Vibrios, Alteromonadales and the SAR11 and SAR116 clades, ISME J 7, 581-591.

[46] Sowell, S. M., Wilhelm, L. J., Norbeck, A. D., Lipton, M. S., Nicora, C. D., Barofsky, D. F., Carlson, C. A., Smith, R. D., and Giovanonni, S. J. (2009) Transport functions dominate the SAR11 metaproteome at low-nutrient extremes in the Sargasso Sea, ISME J 3, 93-105.

[47] Reisch, C. R., Moran, M. A., and Whitman, W. B. (2008) Dimethylsulfoniopropionate-dependent demethylase (DmdA) from Pelagibacter ubique and Silicibacter pomeroyi, J. Bacteriol. 190, 8018-8024.

[48] Yun, D., Dey, M., Higgins, L. J., Yan, F., Liu, H. W., and Drennan, C. L. (2011) Structural basis of regiospecificity of a mononuclear iron enzyme in antibiotic fosfomycin biosynthesis, J. Am. Chem. Soc. 133, 11262-11269.

[49] Zheng, H., Chruszcz, M., Lasota, P., Lebioda, L., and Minor, W. (2008) Data mining of metal ion environments present in protein structures, J. Inorg. Biochem. 102, 1765-1776.

[50] Harding, M. M., Nowicki, M. W., and Walkinshaw, M. D. (2010) Metals in protein structures: a review of their principal features, Crystallography Reviews 16, 247-302.

[51] Alcolombri, U., Elias, M., Vardi, A., and Tawfik, D. S. (2014) Ambiguous evidence for assigning DddQ as a dimethylsulfoniopropionate lyase and oceanic dimethylsulfide producer, Proc. Natl. Acad. Sci. U. S. A. 111, E2078-2079.

[52] Schnitter, R., Gallego, D., and Kersting, B. (2014) Selective complexation of alphaamino acids and simple peptides via their carboxylate groups, Dalton Trans 43, 1363713648. 
[53] Franks, M., Gadzhieva, A., Ghandhi, L., Murrell, D., Blake, A. J., Davies, E. S., Lewis, W., Moro, F., McMaster, J., and Schroder, M. (2013) Five coordinate M(II)diphenolate $[\mathrm{M}=\mathrm{Zn}(\mathrm{II}), \mathrm{Ni}(\mathrm{II})$, and $\mathrm{Cu}(\mathrm{II})]$ Schiff base complexes exhibiting metal- and ligand-based redox chemistry, Inorg. Chem. 52, 660-670.

[54] Kaluarachchi, H., Sutherland, D. E. K., Young, A., Pickering, I.J., Stillman, A.J., and Zamble, D. B. (2009) The Ni(II)-Binding Properties of the Metallochaperone SlyD. J. Am. Chem. Soc. 131, 18489-18500.

[55] Douglas, C. D., Dias, A. V., and Zamble, D. B. (2012) Synthesis and reactivity of 2azametallacyclobutanes, Dalton Trans 41, 7876-7878.

[56] Shimazaki, Y., Huth, S., Karasawa, S., Hirota, S., Naruta, Y., and Yamauchi, O. (2004) Nickel(II)-Phenoxyl Radical Complexes: Structure-Radical Stability Relationship, Inorg. Chem. 43, 7816-7822.

[57] Arora, H., Philouze, C., Jarjayes, O., and Thomas, F. (2010) Co(II), Ni(II), Cu(II) and $\mathrm{Zn}$ (II) complexes of a bipyridine bis-phenol conjugate: generation and properties of coordinated radical species, Dalton Trans 39, 10088-10098.

[58] Lipscomb, W. N., and Strater, N. (1996) Recent Advances in Zinc Enzymology, Chem. Rev. 96, 2375-2434.

[59] Park, H. I., and Ming, L.-J. (1998) The mechanistic role of the coordinated tyrosine in astacin, J. Inorg. Biochem. 72, 57-62.

[60] Boer, J. L., Mulrooney, S. B., and Hausinger, R. P. (2014) Nickel-dependent metalloenzymes, Arch. Biochem. Biophys. 544, 142-152. 


\section{For Table of Contents Use Only.}

Structural and Biochemical Insights into Dimethylsulfoniopropionate Cleavage by Cofactorbound DddK from the Prolific Marine Bacterium

Authors: Nicholas J. Schnicker, Saumya M. De Silva, Jonathan D. Todd, and Mishtu Dey

\section{TOC Graphic.}

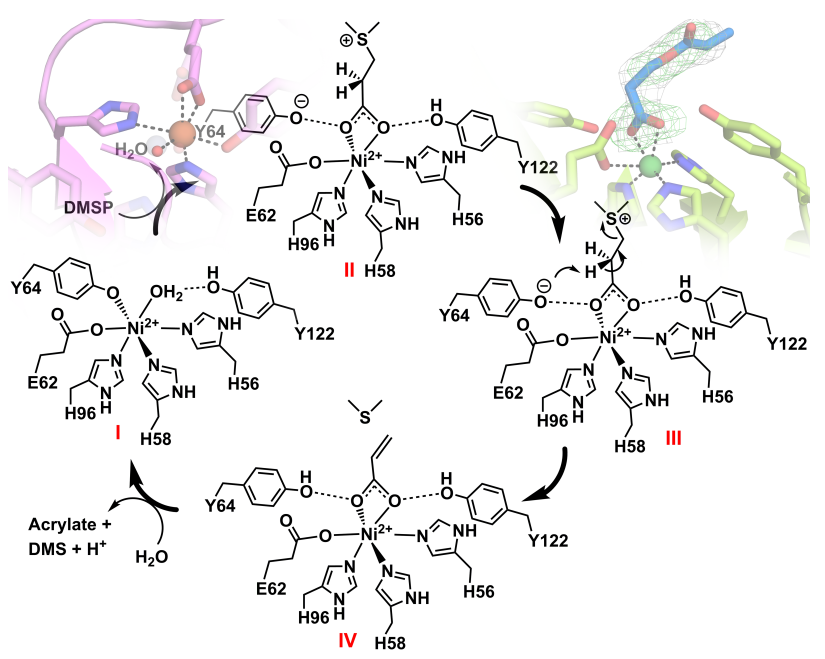

